\title{
ELEMENTAL COMPOSITION AND ENERGY SPECTRA OF GALACTIC COSMIC RAYS DURING SOLAR
} CYCLE 23

\author{
J. S. George ${ }^{1,5}$, K. A. LAve $^{2}$, M. E. Wiedenbeck ${ }^{3}$, W. R. Binns ${ }^{2}$, A. C. Cummings ${ }^{1}$, A. J. Davis ${ }^{1}$, G. A. De Nolfo ${ }^{4}$, \\ P. L. Hink ${ }^{2,6}$, M. H. Israel ${ }^{2}$, R. A. Leske ${ }^{1}$, R. A. MewaldT ${ }^{1}$, L. M. Scott ${ }^{2,7}$, E. C. Stone ${ }^{1}$, T. T. von Rosenvinge ${ }^{4}$, AND \\ N. E. YANASAK ${ }^{3,8}$ \\ ${ }^{1}$ California Institute of Technology, Pasadena, CA 91125, USA \\ ${ }^{2}$ Department of Physics, Washington University in St. Louis, St. Louis, MO 63130, USA \\ ${ }^{3}$ Jet Propulsion Laboratory, California Institute of Technology, Pasadena, CA 91109, USA \\ ${ }^{4}$ NASA Goddard Space Flight Center, Greenbelt, MD 20771, USA \\ Received 2008 October 10; accepted 2009 April 16; published 2009 June 3
}

\begin{abstract}
We report improved measurements of elemental abundances and spectra for galactic cosmic-ray (GCR) nuclei obtained by the Cosmic Ray Isotope Spectrometer on board NASA's Advanced Composition Explorer (ACE) spacecraft during the minimum and maximum phases of solar cycle 23. We discuss results for particles with nuclear charge $5 \leqslant Z \leqslant 28$ and typical energies between 50 and $500 \mathrm{MeV}$ nucleon $^{-1}$. We demonstrate that a detailed "leaky box" Galactic propagation model combined with a spherically symmetric solar modulation model gives a good (but not perfect) fit to the observed spectra by using a solar modulation parameter of $\phi=325 \mathrm{MV}$ at solar minimum and $\phi=900 \mathrm{MV}$ at solar maximum. Although our results are generally consistent with previous measurements from space-based and balloon-based missions, there are significant differences. The large geometrical acceptance and excellent charge resolution of the instrument result in the most detailed and statistically significant record of GCR composition to date in this energy range. The measurements reported here serve as a high-precision baseline for continued studies of GCR composition, solar modulation over the solar cycle, space radiation hazards, and other applications.
\end{abstract}

Key words: cosmic rays - Galaxy: abundances - Sun: activity

Online-only material: color figure

\section{INTRODUCTION}

Galactic cosmic-ray (GCR) nuclei arriving near Earth provide direct access to a sample of material from outside the solar system. The composition and energy spectra of these nuclei reflect the nucleosynthetic processes at work in the source, as well as the conditions that the material experienced during acceleration and transport through the Galaxy. In addition, the energy spectra of ions entering the heliosphere are distorted by interactions with a turbulent magnetic field carried outward by an expanding solar wind. While we cannot directly observe the ions outside the heliosphere, measurements of the local spectra, particularly during the minimum phase of solar activity, can provide information about the interstellar spectra. Comparisons with solar maximum data shed light on heliospheric processes.

The Cosmic Ray Isotope Spectrometer (CRIS; Stone et al. 1998) on board NASA's Advanced Composition Explorer (ACE) spacecraft has provided detailed measurements of the properties of cosmic rays from its halo orbit about the L1 Lagrange point $1.5 \times 10^{6} \mathrm{~km}$ sunward of Earth since its launch in 1997 August. The large geometrical acceptance and excellent charge and mass resolution of the instrument have enabled the compilation of the most detailed and statistically significant records of GCR composition to date. We report here on measurements of elemental composition and spectra for GCR nuclei with

\footnotetext{
5 Now at Space Sciences Department, The Aerospace Corporation, Los Angeles, CA 90009, USA.

6 Now at Burle Industries, Inc., Lancaster, PA 17601, USA.

7 Now at Rutgers, the State University of New Jersey, Piscataway, NJ 08854, USA.

8 Now at Department of Psychology, University of Georgia, Athens, GA 30602, USA.
}

nuclear charge $5 \leqslant Z \leqslant 28$ and energies between 50 and $500 \mathrm{MeV}$ nucleon $^{-1}$ during a period of solar minimum from 1997 August to 1998 April, and a period of solar maximum from 2001 May to 2003 September.

\section{INSTRUMENT DESCRIPTION}

The CRIS instrument consists of four silicon solid-statedetector stacks (see Figure 1). Each stack is composed of nine circular detectors, with six (E3-E8) made of electrically paired silicon wafers. Detectors E2 through E7 include annular guard rings for rejecting particles exiting the sides. Trajectories are measured with a square scintillating optical fiber trajectory (SOFT) hodoscope with three $x-y$ tracking layers. An additional pair of fiber layers serves as a trigger. The charge and mass of particles stopping in the silicon detectors are determined from the energy deposited $\left(E^{\prime}\right.$, in $\left.\mathrm{MeV}\right)$ in the detector in which the particle stops, combined with multiple measurements of the rate of energy loss $\left(\mathrm{d} E / \mathrm{d} x\right.$, in $\left.\mathrm{MeV} \mathrm{g}^{-1} \mathrm{~cm}^{2}\right)$ in the other detectors through which it passed. Details of the CRIS instrument construction and expected performance have been reported previously (Stone et al. 1998).

\section{DATA SELECTION}

A histogram of the calculated nuclear charge for particles stopping in detectors E3 through E8 is shown in Figure 2. The charge peaks are clearly defined for all elements from boron $(Z=5)$ through nickel $(Z=28)$. Calculation of the charge involves an implicit dependence on mass in the form of an assumed charge-to-mass ratio (Stone et al. 1998). Therefore, isotope features are also visible in the charge histogram, for 
Cosmic Ray Isotope Spectrometer (CRIS)

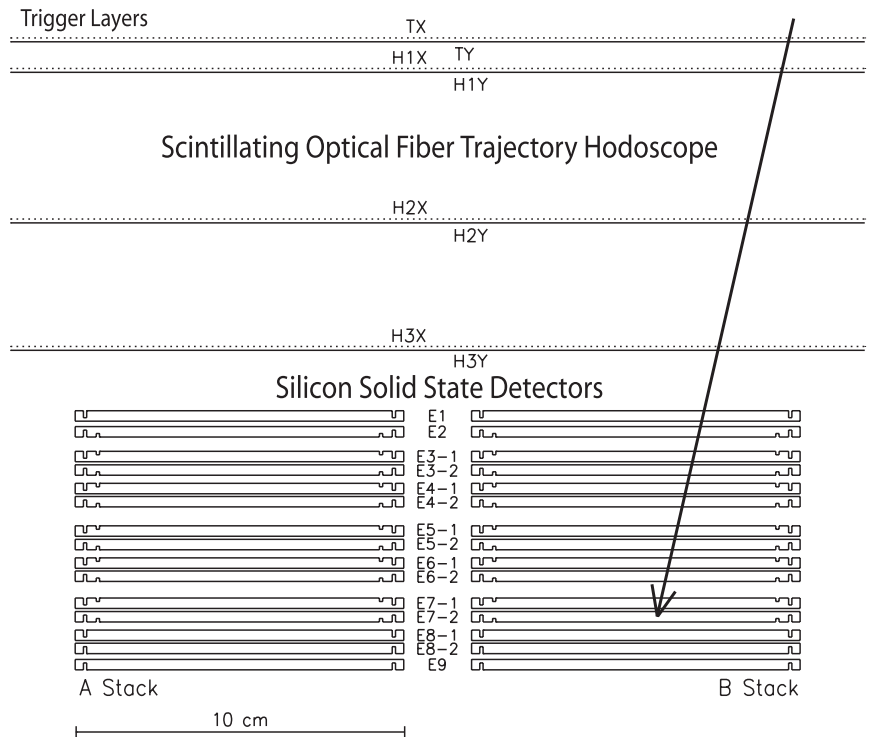

Figure 1. CRIS instrument diagram (two of four circular detector stacks shown). The annular guard rings in the middle silicon detectors (E2-E7) tag particles exiting the sides. Particle trajectories are measured with three $x-y$ layers of scintillating optical fibers, while a fourth layer at the top of the instrument provides a trigger pulse for the system. The charge and mass are determined from multiple measurements of the energy loss $\left(\mathrm{MeV} \mathrm{g}^{-1} \mathrm{~cm}^{2}\right)$ and total deposited energy $(\mathrm{MeV})$ for particles that stop in the detectors. The arrow represents the trajectory of a particle that stops in the bottom wafer of detector E7.

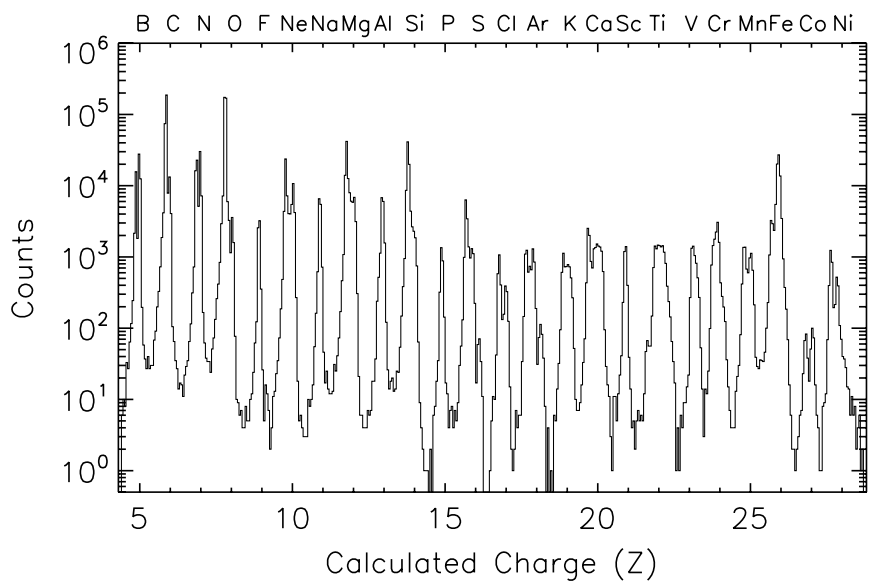

Figure 2. Calculated nuclear charge $(Z)$ histograms for boron $(Z=5)$ to nickel $(Z=28)$ stopping in detectors E3 through E8, including data taken from 1997 August 28 to 1998 April 19 and 2001 May 1 to 2003 September 1. Elements are clearly separated, and isotope features are also visible. The data have been subjected to the selection and quality criteria described in detail in Section 3.

example, note the structure of neon $(Z=10)$ or calcium $(Z=20)$.

The CRIS instrument has been operating for a complete solar cycle. The data include significant periods during both solar minimum and solar maximum conditions. Figure 3 shows the neutron monitor levels from the Climax station averaged over each Bartels rotation. Also shown are the time periods during which various other experiments were operating. Three of these are balloon instruments: a University of Alabama in Huntsville (UAH) experiment (Derrickson et al. 1992), the CRISIS detector from the University of Minnesota (Young et al. 1981), and a series of flights from the University of New Hampshire (UNH; Lezniak \& Webber 1978). The filled portions of the bars in

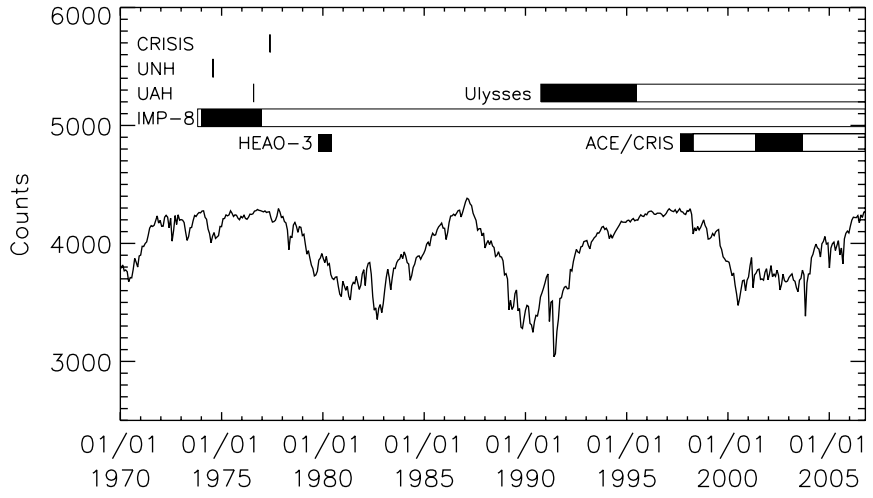

Figure 3. Neutron monitor levels from the Climax station, averaged over each Bartels rotation. The bars above the data show time periods during which various experiments have been operating. The filled sections indicate periods used for comparison in this paper. The two filled periods for $A C E / C R I S$ represent the solar minimum and solar maximum periods used in this paper. Previous experiments include three space missions, IMP-8 (Garcia-Muñoz et al. 1977b), Ulysses (DuVernois \& Thayer 1996), and HEAO-3 (Engelmann et al. 1990), and three balloon experiments, the University of Alabama (Huntsville; Derrickson et al. 1992), the University of New Hampshire (Lezniak \& Webber 1978), and CRISIS (Young et al. 1981).

Figure 3 indicate the time periods of data used for comparisons in this study. The two filled sections in the bar for $A C E /$ CRIS represent the solar minimum and solar maximum periods considered here. From the Ulysses mission, relative abundances have been published (DuVernois \& Thayer 1996) only for the indicated period. The $A C E$ spacecraft and instruments continue to operate nominally with enough onboard resources to support the mission well beyond the next solar maximum.

Particle events are sorted onboard the spacecraft into prioritized buffers based on loose charge estimates $(\mathrm{H}, \mathrm{He}$, or $Z>2)$ and trajectory quality criteria. Only a fraction of the highly abundant hydrogen and helium events are recorded to ensure that the pulse heights for rarer, heavier $(Z>2)$ species are sent to the ground. For events to be treated as valid, we require that the particles have penetrated into the second detector (E2) to ensure at least one measurement of energy loss $(\mathrm{d} E / \mathrm{d} x)$ and one of the residual energy $\left(E^{\prime}\right)$ in the stopping detector. Data for the solar minimum period were obtained during 219 days selected from a 236 day period from 1997 August 28 through 1998 April 19. A large solar energetic particle event beginning on 1998 April 20 accompanied by a Forbush decrease marked the end of solar minimum conditions. Data for the solar maximum period were selected from 733 days during an 854 day period from 2001 May 1 to 2003 September 1. For more information about the time selection criteria, see Appendix A.4.

Valid reconstructed trajectories were required to pass at least $0.5 \mathrm{~mm}$ inside any edge of the top SOFT trigger plane and also $0.5 \mathrm{~mm}$ radially inside the active areas of the top two detectors, E1 and E2. The same margin condition was applied to the projection of the track onto the top face of the detector following that in which the particle stopped. Additionally, due to a difference in detector size, special margin conditions were needed for particles that penetrated the seventh (E7) or eighth (E8) detectors. Valid trajectories were required to pass $0.5 \mathrm{~mm}$ radially inside the bottom face of E7 and also project within E7's radius at the top of E8. The absence of high-level signals in the annular guard rings and the last (E9) detector ensured the selection of particles stopping inside the stack. Incident angles relative to the detector normal were restricted to a maximum of $30^{\circ}$. 


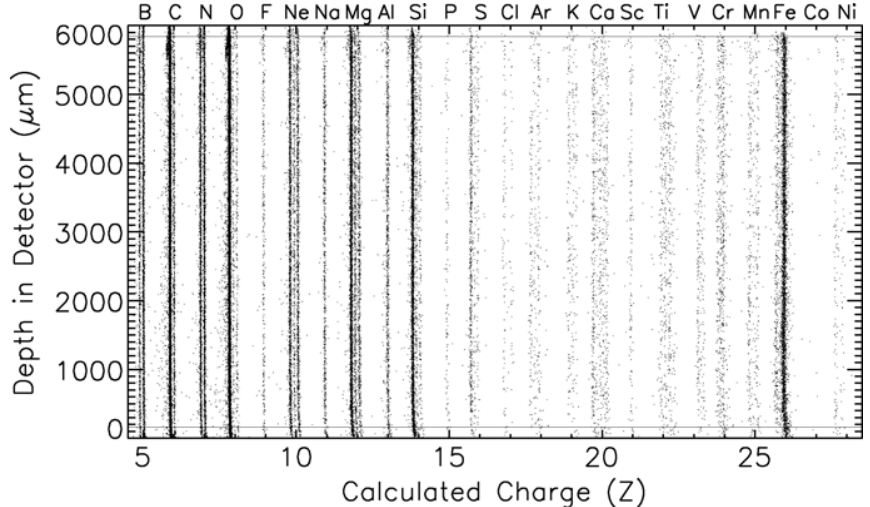

Figure 4. Calculated nuclear charge $(Z)$ as a function of calculated stopping depth for particles stopping in the E3 detectors. The horizontal lines indicate the location of $160 \mu \mathrm{m}$ cuts from the detector faces. Element tracks are fully separated and prominent isotope tracks are also clearly visible.

For particles stopping in detectors E2-E8, the charge and mass were calculated using the energy loss in each detector and the total path length through all penetrated detectors prior to the stop detector, corrected for the angle of incidence (Stone et al. 1998). With the charge, mass, and energy loss in the stop detector, the particle's depth in the stop detector was calculated using the semiempirical range-energy relations for hydrogen from Andersen \& Ziegler (1977). The relations were scaled to heavy ions using an expression for the effective charge of energetic heavy ions obtained from Ahlen (1980). Particles calculated to have stopped within $160 \mu \mathrm{m}$ of the top or bottom face of each detector were rejected (see Figure 4) to avoid small tails in the calculated charge distributions due to thin $(\sim 60 \mu \mathrm{m})$ "dead layers" where the charge collection is not fully efficient.

\section{ELEMENTAL SPECTRA}

Elemental spectra were obtained after correcting for geometrical acceptance, live time, detector efficiencies, and energy intervals. The spectra for solar minimum are plotted in Figure 5 with arbitrary scale factors to allow easy comparison of the shapes. Corresponding curves for solar maximum are given in Figure 6. The data shown in these figures are also listed in Tables B1 and B2 in Appendix B.

The statistical contribution to the intensity uncertainties is typically very small for all but the rarest elements, ranging from $0.6 \%$ for oxygen in each range to $\sim 10 \%$ for phosphorus and scandium during the solar minimum period. Only the cobalt points have significantly larger statistical uncertainties $(25 \%)$. Statistics during solar maximum are comparable or better in spite of the lower intensities, due to the longer time period used. Systematic uncertainties arise from the calculation of the intensity correction factors. Principal contributions come from the geometry factor $(2 \%)$, the correction for spallation in the instrument $(\sim 1 \%-5 \%$, depending on the charge and range), and the determination of the SOFT efficiency (2\%). Errors quoted in Tables B1 and B2 are the quadratic sum of the statistical and systematic contributions.

The solid curves in Figures 5 and 6 are quadratic fits in $\log$ (Intensity) versus $\log$ (Energy/nucleon) to the seven data points of the energy spectra for each element. The dashed curves are the result of a steady-state leaky box propagation calculation described in greater detail in Appendix C.1. The model includes the effects of galactic transport, including fragmentation of heavier nuclei, radioactive decays, energy losses, and escape

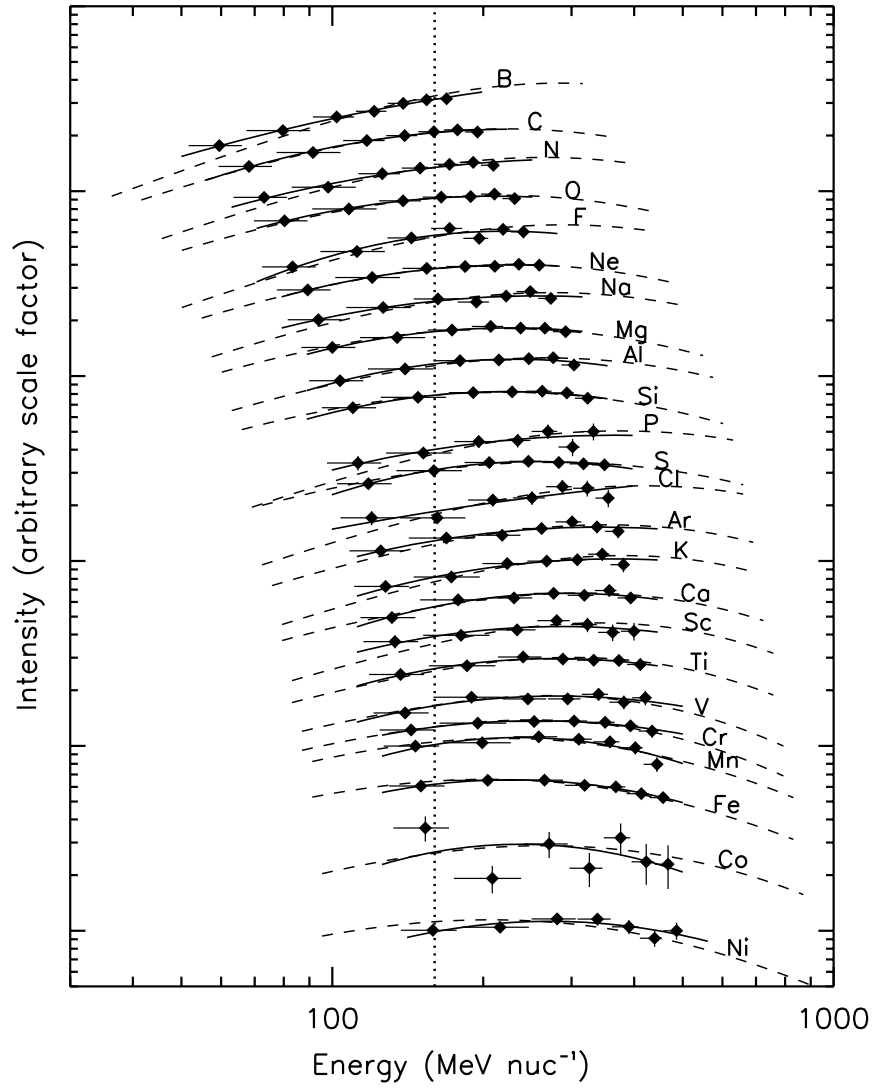

Figure 5. CRIS elemental GCR spectra during solar minimum. Arbitrary scale factors have been applied to the intensity of each element for presentation of the spectral shapes. The dashed curves are the result of a cosmic-ray propagation model calculation. The solid curves show the fits used to determine relative abundances. The dotted line at $160 \mathrm{MeV}$ nucleon $^{-1}$ shows where relative abundances are reported (Table 1).

from the Galaxy. Reacceleration of nuclei by multiple shocks is not included. Modulation of the spectra inside the heliosphere by interactions with the solar wind is based on a spherically symmetric Fisk model (Fisk 1971). Although we have used the Fisk numerical solution in our fits and not the force field solution introduced by Gleeson \& Axford (1968), for convenience we have characterized our fits by the modulation parameter $\phi$, as described in Appendix C.2. We fit the data with a model that corresponds to an effective modulation parameter $\phi=325 \mathrm{MV}$ for our solar minimum period and $\phi=900 \mathrm{MV}$ for our solar maximum period. These particular values will not necessarily correspond to those determined from fitting these or other data with other modulation models or with different interstellar spectra (see, for example, Usoskin et al. 2005, and references therein).

The model spectra were obtained by summing the calculated isotopic spectra for each element. For species whose locally measured abundances contain a significant primary contribution, the source abundances were adjusted to improve agreement with the data. Good agreement was obtained for most elements in the solar minimum data set.

\section{COMPOSITION}

Relative GCR abundances at $160 \mathrm{MeV}$ nucleon $^{-1}$ for the CRIS solar minimum and maximum periods are given in Table 1, normalized to $\mathrm{Si} \equiv 1000$. We note that the intensity of silicon at $160 \mathrm{MeV}$ nucleon $^{-1}$ during solar maximum is lower by a 


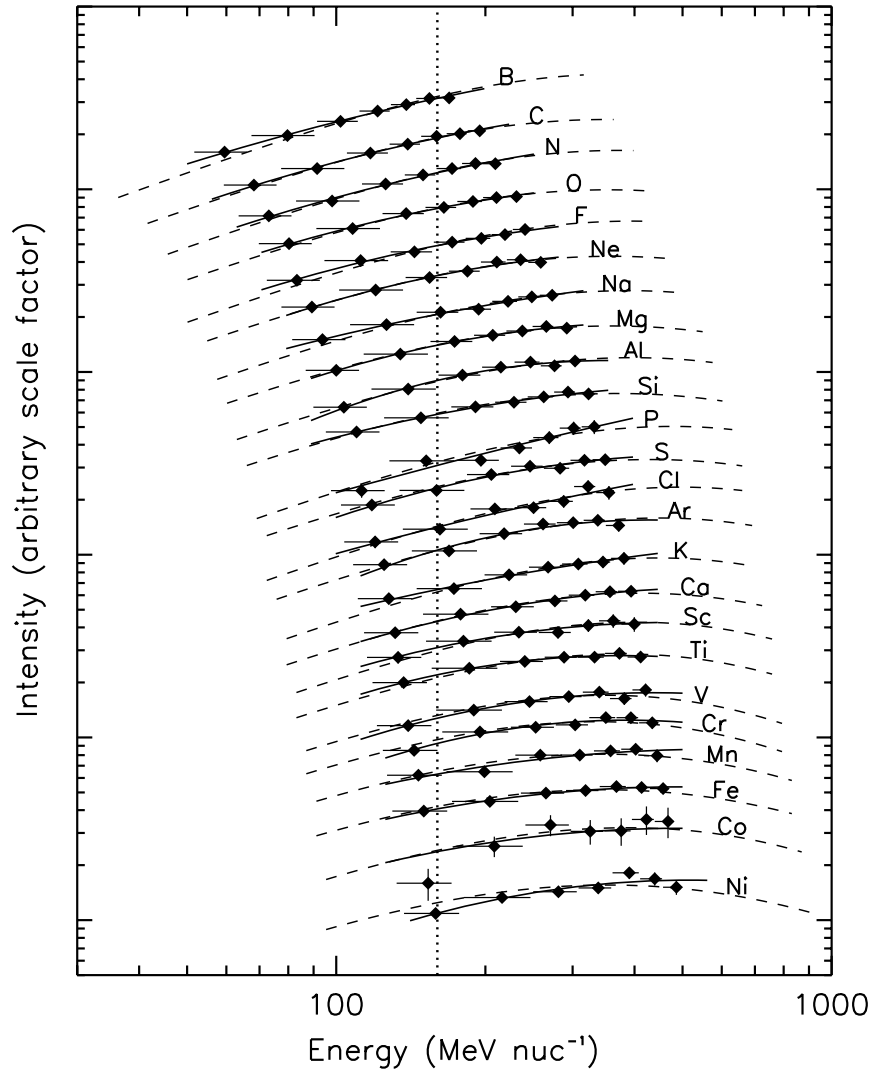

Figure 6. CRIS elemental GCR spectra during solar maximum. Arbitrary scale factors have been applied to the intensity of each element for presentation of the spectral shapes. The dashed curves are the result of a cosmic-ray propagation model calculation. The solid curves show the fits used to determine relative abundances. The dotted line at $160 \mathrm{MeV}$ nucleon ${ }^{-1}$ shows where relative abundances are reported (Table 1).

factor of 0.27 than that measured at solar minimum, allowing for a comparison of the absolute intensity levels in the two time periods. The composition is energy dependent, and this energy was chosen because the CRIS sensitivity for all species between boron and nickel overlaps at this point.

The relative abundances were determined by individually fitting each spectrum of seven intensity data points with a parabola in $\log$ (Intensity) versus $\log$ (Energy/nucleon). Cobalt did not have sufficient statistics for a good fit, so manganese was used as a template for the shape and only the overall normalization was fitted. The results of the fits are indicated by the solid curves in Figures 5 and 6. The relative abundances were taken from the ratios of the fit curves at $160 \mathrm{MeV}$ nucleon $^{-1}$. The uncertainties in the relative abundances were taken to be similar to those of the data themselves, with a statistical contribution based on the total number of counts. The residual systematic uncertainties will tend to cancel when comparing adjacent elements. In most cases, the statistical uncertainty is much smaller than the systematic contribution.

Our observed GCR abundances for solar minimum are plotted in Figure 7, supplemented with GCR observations for $Z<5$ reported elsewhere (see Wang et al. 2002; de Nolfo et al. 2006). The data are given at $160 \mathrm{MeV}$ nucleon ${ }^{-1}$ and are normalized to $\mathrm{Si} \equiv 1000$. These abundances are compared with solar system abundances given by Lodders (2003). The odd- $Z$ heavy nuclei, as well as a few notable even- $Z$ nuclei $(\mathrm{Be}, \mathrm{Ca}, \mathrm{Ti}$, and $\mathrm{Cr}$ ), show significant GCR overabundances. This well known property of cosmic-ray abundances demonstrates the effect of
Table 1

CRIS Relative Elemental Abundances at $160 \mathrm{MeV}$ Nucleon $^{-1}$

\begin{tabular}{lcr}
\hline \hline Element & Solar Minimum & Solar Maximum \\
\hline $\mathrm{B}$ & $1803.8 \pm 10.4$ & $1986.4 \pm 11.3$ \\
$\mathrm{C}$ & $7337.0 \pm 18.4$ & $6780.2 \pm 18.4$ \\
$\mathrm{~N}$ & $1713.7 \pm 8.4$ & $1836.1 \pm 9.0$ \\
$\mathrm{O}$ & $7082.6 \pm 16.0$ & $6520.6 \pm 15.6$ \\
$\mathrm{~F}$ & $101.8 \pm 1.9$ & $123.6 \pm 2.1$ \\
$\mathrm{Ne}$ & $998.7 \pm 5.6$ & $1050.4 \pm 5.8$ \\
$\mathrm{Na}$ & $189.6 \pm 2.4$ & $211.5 \pm 2.5$ \\
$\mathrm{Mg}$ & $1368.2 \pm 6.1$ & $1367.3 \pm 6.0$ \\
$\mathrm{Al}$ & $202.7 \pm 2.3$ & $226.3 \pm 2.4$ \\
$\mathrm{Si}$ & $1000.0 \pm 5.0$ & $1000.0 \pm 4.8$ \\
$\mathrm{P}$ & $26.2 \pm 0.8$ & $34.2 \pm 0.8$ \\
$\mathrm{~S}$ & $157.0 \pm 1.9$ & $181.2 \pm 1.9$ \\
$\mathrm{Cl}$ & $24.9 \pm 0.7$ & $38.4 \pm 0.9$ \\
$\mathrm{Ar}$ & $58.8 \pm 1.1$ & $78.5 \pm 1.2$ \\
$\mathrm{~K}$ & $41.6 \pm 0.9$ & $62.5 \pm 1.1$ \\
$\mathrm{Ca}$ & $124.8 \pm 1.5$ & $155.8 \pm 1.6$ \\
$\mathrm{Sc}$ & $26.0 \pm 0.7$ & $35.2 \pm 0.8$ \\
$\mathrm{Ti}$ & $100.4 \pm 1.4$ & $125.6 \pm 1.5$ \\
$\mathrm{~V}$ & $45.7 \pm 0.9$ & $54.7 \pm 0.9$ \\
$\mathrm{Cr}$ & $98.8 \pm 1.3$ & $109.7 \pm 1.3$ \\
$\mathrm{Mn}$ & $61.4 \pm 1.1$ & $71.4 \pm 1.1$ \\
$\mathrm{Fe}$ & $653.7 \pm 3.5$ & $742.1 \pm 3.4$ \\
$\mathrm{Co}$ & $3.7 \pm 0.3$ & $4.6 \pm 0.3$ \\
$\mathrm{Ni}$ & $27.8 \pm 0.7$ & $33.8 \pm 0.7$ \\
\hline
\end{tabular}

Notes. Values are normalized to Si. Only the statistical uncertainties are given. The absolute intensity for silicon at $160 \mathrm{MeV}$ nucleon $^{-1}$ is $(107.4 \pm 3.3) \times$ $10^{-9}\left(\mathrm{~cm}^{2} \mathrm{~s} \mathrm{sr} \mathrm{MeV} \text { nucleon }{ }^{-1}\right)^{-1}$ for solar minimum and $(29.1 \pm 0.9) \times 10^{-9}$ $\left(\mathrm{cm}^{2} \mathrm{~s} \mathrm{sr} \mathrm{MeV} \mathrm{nucleon}^{-1}\right)^{-1}$ for solar maximum.

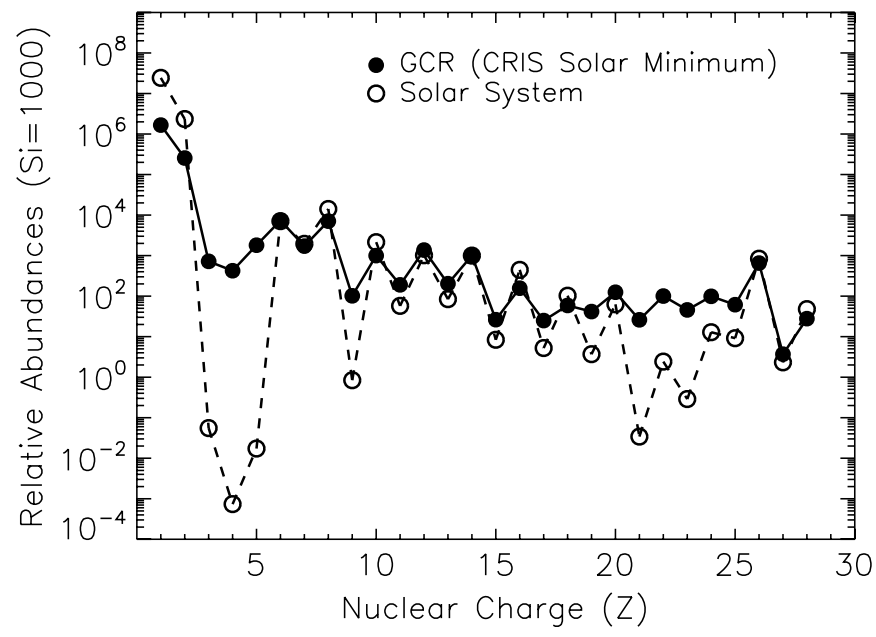

Figure 7. Comparison of GCR solar minimum abundances (filled circles) with solar system abundances (open circles). The CRIS solar minimum results reported in this paper (Table 1 ) are used for the $Z \geqslant 5$ GCR abundances. For $Z<5$, the GCR data come from Wang et al. (2002) and de Nolfo et al. (2006). The solar system abundances are taken from Lodders (2003).

(A color version of this figure is available in the online journal.)

the interstellar fragmentation of heavier elements into secondary cosmic rays, which fills in the abundances of the rarer elements.

\section{DISCUSSION}

\subsection{Solar Minimum Primary-element Spectra}

Figure 8 shows selected CRIS primary-element spectra during the 1997-1998 solar minimum period. Spectra from various experiments during the 1976-1978 minimum are plotted for 


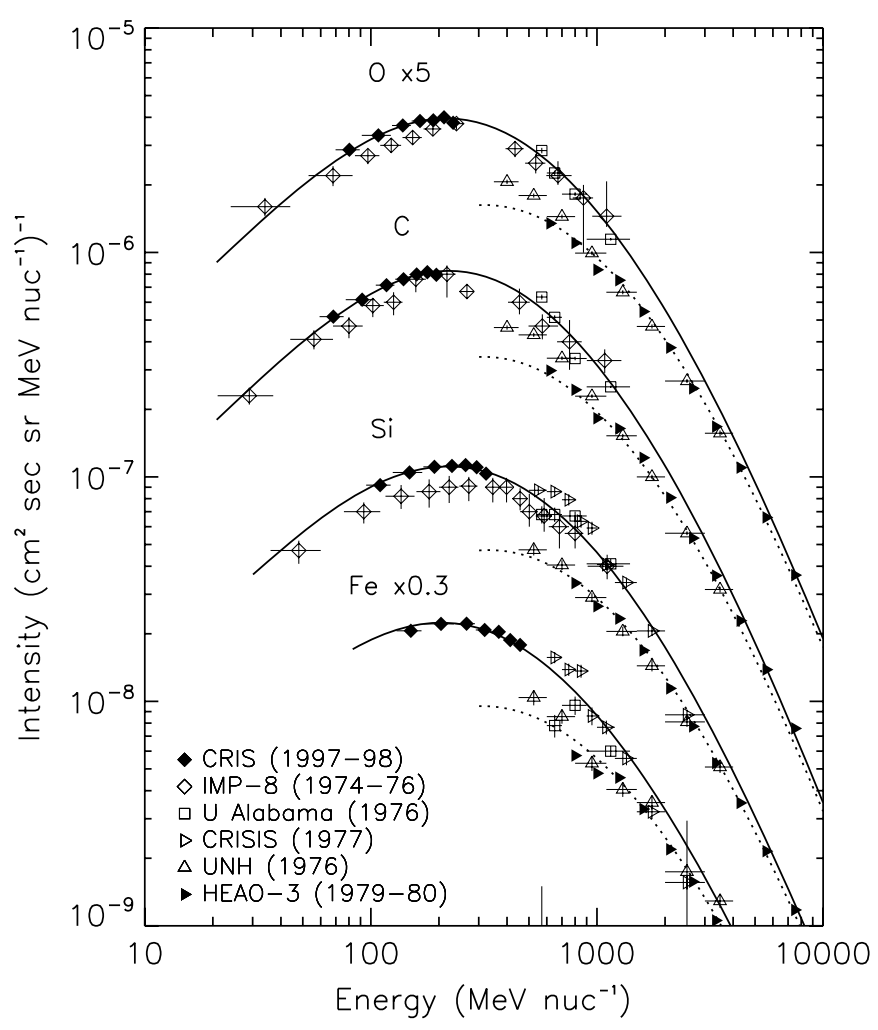

Figure 8. Observed spectra for selected elements, including the CRIS solar minimum data. For references to earlier measurements, see the caption to Figure 3. The solid curves are the result of a propagation model calculation corresponding to a solar modulation level of $\phi=325 \mathrm{MV}$. The dotted curves correspond to $\phi=750 \mathrm{MV}$.

comparison. Spectra for the elements in this paper are not available for the 1986-1987 solar minimum.

The CRIS data recorded during 1997-1998 are probably the most representative of true solar minimum conditions available. The typical neutron monitor levels experienced in 1997-1998 (see Figure 3) are similar to those experienced during the period in which the Interplanetary Monitoring Platform (IMP)8 spacecraft (Garcia-Muñoz et al. 1977b) made measurements in the 1974-1976 minimum, though the IMP-8 period included a large neutron monitor decrease observed around the beginning of 1975 (see also Davis et al. 2001a). In general, the CRIS and $I M P-8$ spectra, which cover similar energy intervals, are in reasonable agreement, allowing for the differences in average solar modulation level. Both are consistent with higher energy balloon measurements, which are less affected by variations in the solar cycle but require corrections for the atmospheric overburden. The UNH data (Lezniak \& Webber 1978) were taken well into the large intensity decrease of 1975 and should be expected to have a solar modulation level midway between that for CRIS solar minimum and CRIS solar maximum.

Also plotted in Figure 8 are results from the French-Danish experiment on board the $H E A O-3$ spacecraft (Engelmann et al. 1990). Although HEAO-3 did not fly during the solar minimum period, the high statistical accuracy of its data make it the canonical database for comparison at higher energies. Furthermore, solar modulation has less of an effect on the observed spectra at higher energies so the HEAO-3 data provide a basis for estimating the intensity and spectra of elements just outside the heliosphere.

The propagation model curves overplotted on Figure 8 allow for a comparison between the CRIS and $H E A O-3$ data. The solid

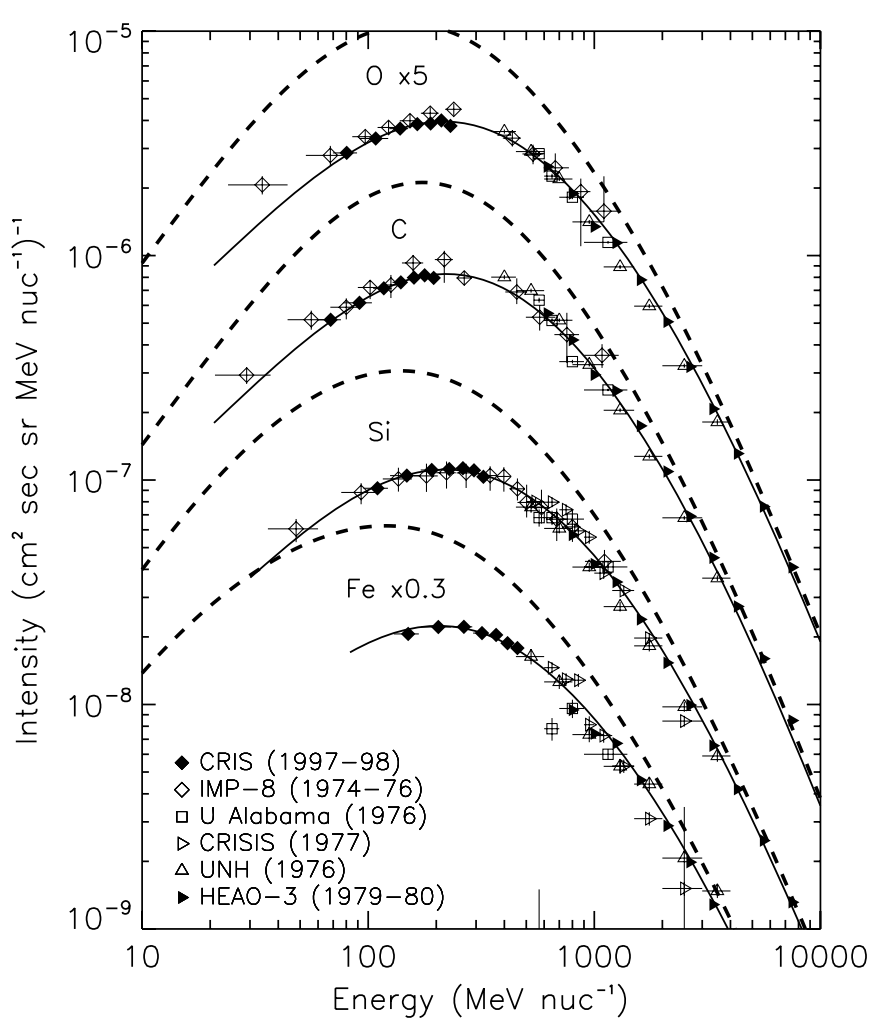

Figure 9. Observed spectra for selected elements, including the CRIS solar minimum data. The data from previous experiments have been adjusted (see the text) to the CRIS solar modulation level (corresponding to $\phi=$ $325 \mathrm{MV}$ ). For references to earlier measurements, see the caption to Figure 3. The corresponding calculated interstellar spectra from the propagation model described in Appendix C. 1 are indicated by the dashed lines.

curves show the model spectra calculated with a modulation parameter corresponding to $\phi=325 \mathrm{MV}$, appropriate for the 1997-98 minimum period. The dotted lines show the same model spectra corresponding to a modulation of $\phi=750 \mathrm{MV}$, more appropriate for the average conditions under which the HEAO-3 data were taken. It is clear that both sets of data are fitted very well by the same interstellar abundances and spectra. The observed differences at Earth can be accounted for solely by differences in the level of solar modulation.

Figure 9 shows the same solar minimum spectra with data from prior experiments adjusted to the CRIS solar minimum solar modulation level (corresponding to $\phi=325 \mathrm{MV}$ ). The CRIS and HEAO-3 experiments were assigned levels of $325 \mathrm{MV}$ and $750 \mathrm{MV}$, respectively, based on the success in fitting the data with those parameters. Values for other experiments were found by linearly interpolating the mean Climax neutron monitor rates during the periods data were taken. The following solar modulation levels were used: $275 \mathrm{MV}$ (CRISIS), $325 \mathrm{MV}$ (UAH), $400 \mathrm{MV}(I M P-8)$, and $625 \mathrm{MV}$ (UNH). Ratios of the propagation model curves using the CRIS modulation level and the derived modulation level for each experiment gave an energy-dependent correction, which was applied to the data. When adjusted in this way, all of the data are consistent with the CRIS solar minimum spectra. Such solar minimum data are relevant for studies of radiation exposure in space and radiation doses for astronauts (Davis et al. 2001a; Mewaldt et al. 2005; O'Neill 2006). It would be of interest to extend the analysis to the approaching solar maximum. Also shown in this figure are the calculated interstellar spectra (dashed lines) from the propagation model described in Appendix C. 1 for the given 


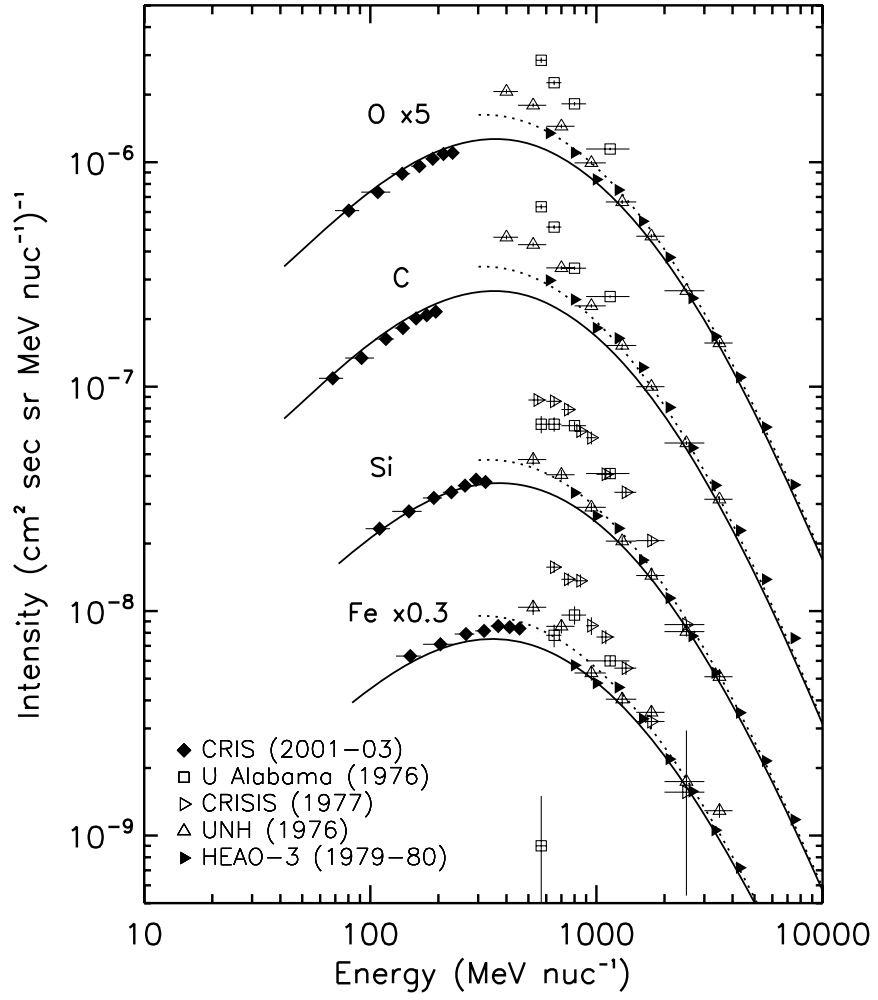

Figure 10. Observed spectra for selected elements, including the CRIS solar maximum data. For references to earlier measurements, see the caption to Figure 3. The solid curves are the result of a propagation model calculation using a solar modulation parameter corresponding to $\phi=900 \mathrm{MV}$. The dotted curves correspond to $\phi=750 \mathrm{MV}$.

elements. Appendix C.1 also includes analytical approximations to these spectra.

\subsection{Solar Maximum Primary-element Spectra}

After a transition period from early 1998 to the beginning of 2001, the mean neutron monitor rate from the Climax station (Figure 3 ) was relatively stable at a level roughly $15 \%$ below that observed during the solar minimum period. We have taken a 28 month period from 2001 May 1 through 2003 September 1 as a sample of solar maximum conditions. Based on the same interpolation of mean neutron monitor rates described for other experiments above, we infer an average modulation parameter corresponding to $\phi=900 \mathrm{MV}$ for this solar maximum period.

Figure 10 shows the comparison between previously collected data and those obtained from CRIS during solar maximum. The CRIS solar maximum intensities are reduced by more than a factor of 3 compared with solar minimum and the spectra are much more consistent with the HEAO-3 spectra in both shape and absolute level. The solid curves give the result of a propagation model calculation corresponding to a modulation parameter of $\phi=900 \mathrm{MV}$. The dotted curve, as before, shows the same model modulated corresponding to $\phi=750 \mathrm{MV}$. It is clear from the shape of the spectra, as from the neutron monitor data, that the CRIS solar maximum period has a higher average level of modulation than during the HEAO-3 flight.

It is also clear that the same model that fits all CRIS spectra simultaneously during solar minimum conditions does not achieve the same goal during solar maximum. The spectrum plotted for silicon fits the model well, while the oxygen and carbon data fall below the model and the iron data fall above the model. The difficulties in precisely fitting this extensive set

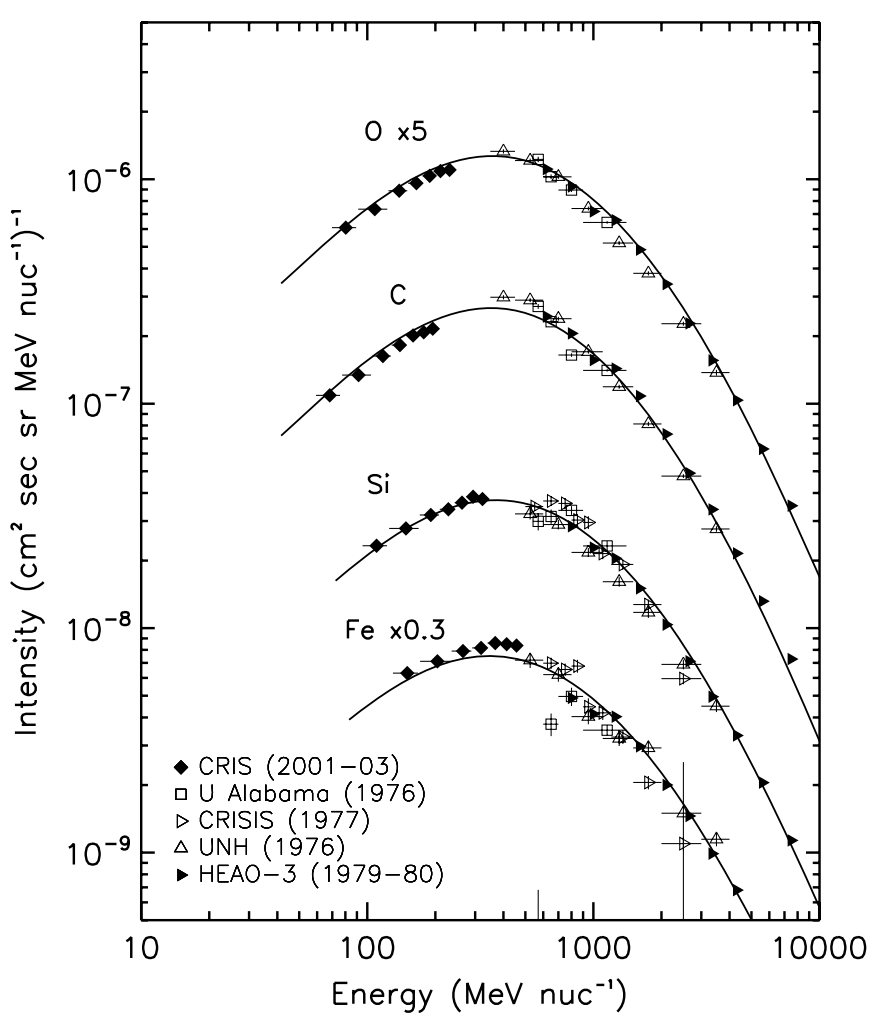

Figure 11. Observed spectra for selected elements, including the CRIS solar maximum data. The data from previous experiments have been adjusted (see the text) to the CRIS solar maximum modulation level (corresponding to $\phi=900 \mathrm{MV}$ ). For references to earlier measurements, see the caption to Figure 3.

of observations are not surprising given the highly simplified solar modulation model we are using, which not only assumes spherical symmetry and the absence of drift effects, but also employs a scattering mean free path with no radial dependence and a rigidity dependence (assumed to be linear, see Section C.2) that does not vary over the solar cycle. These data may thus help constrain models of modulation more sophisticated than the model used here (see, e.g., Potgieter \& Langner 2004).

As previously described in Section 6.1, the propagation model curves appropriate to each experiment's average level of modulation can be used to adjust previous data to the level seen during the CRIS solar maximum period. The result is shown in Figure 11. Here, we also see that the data from other experiments are consistent with the CRIS solar maximum spectra.

\subsection{Secondary Elements}

As a further indication that the model gives consistent results, we show representative secondary/primary ratios, $\mathrm{B} / \mathrm{C},(\mathrm{Cl}+\mathrm{K}) / \mathrm{Fe}$, and $(\mathrm{Sc}+\mathrm{Ti}+\mathrm{V}) / \mathrm{Fe}$, as a function of energy for solar minimum and maximum in Figure 12. The numerators of the ratios reflect the abundance of secondaries produced from fragmentation in the interstellar medium (see Figure 7), while the denominators measure mostly primary material from the source.

The model features a maximum as a function of energy corresponding to the distributions of path lengths traversed in the Galaxy by the cosmic rays we sample at Earth (Davis et al. 2000; Yanasak et al. 2001). The CRIS solar minimum data are at energies below this maximum and so measure secondary contributions that increase with energy. The differences between the solar minimum and maximum ratios are due to the higher inter- 


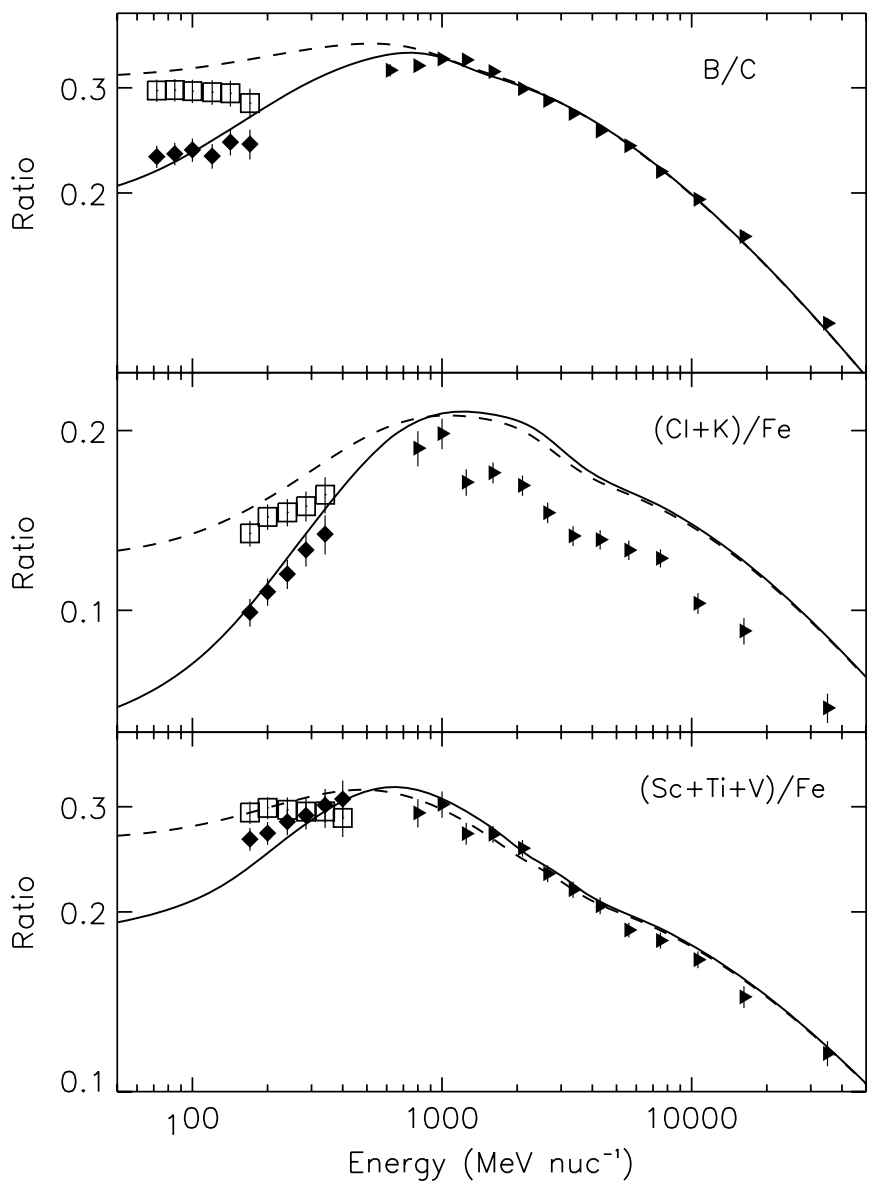

Figure 12. CRIS elemental secondary/primary ratios $\mathrm{B} / \mathrm{C},(\mathrm{Cl}+\mathrm{K}) / \mathrm{Fe}$, and $(\mathrm{Sc}+\mathrm{Ti}+\mathrm{V}) / \mathrm{Fe}$ during solar minimum (solid diamonds) and solar maximum (open squares). Data from $H E A O-3$ at energies greater than $800 \mathrm{MeV}^{\text {nucleon }}{ }^{-1}$ are shown for context (Engelmann et al. 1990). The dashed curves reflect a propagation model calculation corresponding to a solar modulation level of $\phi=900 \mathrm{MV}$, while the solid curves correspond to $\phi=325 \mathrm{MV}$. stellar energies from which solar maximum data are sampled. The HEAO-3 data are generally at energies above the peak and see secondary-to-primary ratios that decrease with energy, due to the easier escape of higher energy particles from the Galaxy. Better fits to the CRIS data may be achieved using a more sophisticated model.

\subsection{Relative Abundances}

A comparison of the CRIS relative abundances for solar minimum and maximum is shown in the top right panel of Figure 13, where the effects of solar modulation are easily seen. Higher modulation levels mean that the arriving particles come, on average, from higher energy particles outside the heliosphere (Niebur et al. 2003). At CRIS energies, higher energies imply longer path lengths and more secondaries. The elements from phosphorus to potassium $(15 \leqslant Z \leqslant 19)$ are produced to a significant extent as tertiary interaction products, and so are even more sensitive to the mean path length than secondary species such as $21 \leqslant Z \leqslant 25$. For this reason, there are obvious overabundances for several elements.

The differences between the CRIS solar minimum composition and measurements by other experiments are seen in Figure 13, where $I M P-8$ and $H E A O-3$ data are plotted as a ratio to the CRIS solar minimum composition. In the top left panel, the IMP-8 abundances agree fairly well with CRIS abundances for even- $Z$ elements. This is expected since data from both instruments correspond to similar solar minimum periods. Odd- $Z$ elements are less abundant than even- $Z$ elements. The higher abundances in IMP-8's odd-Z elements could be the result of spillover from adjacent even- $Z$ elements (see, e.g., Garcia-Muñoz et al. 1977a). In Figure 2, the CRIS peak-to-valley ratio for most elements is better than a factor of 100, and in some cases is better than a factor of 1000, thus greatly reducing spillover contribution as compared with data from earlier instruments.

The bottom panels of Figure 13 display comparisons of the CRIS solar minimum data with $H E A O-3$ data at 1 and

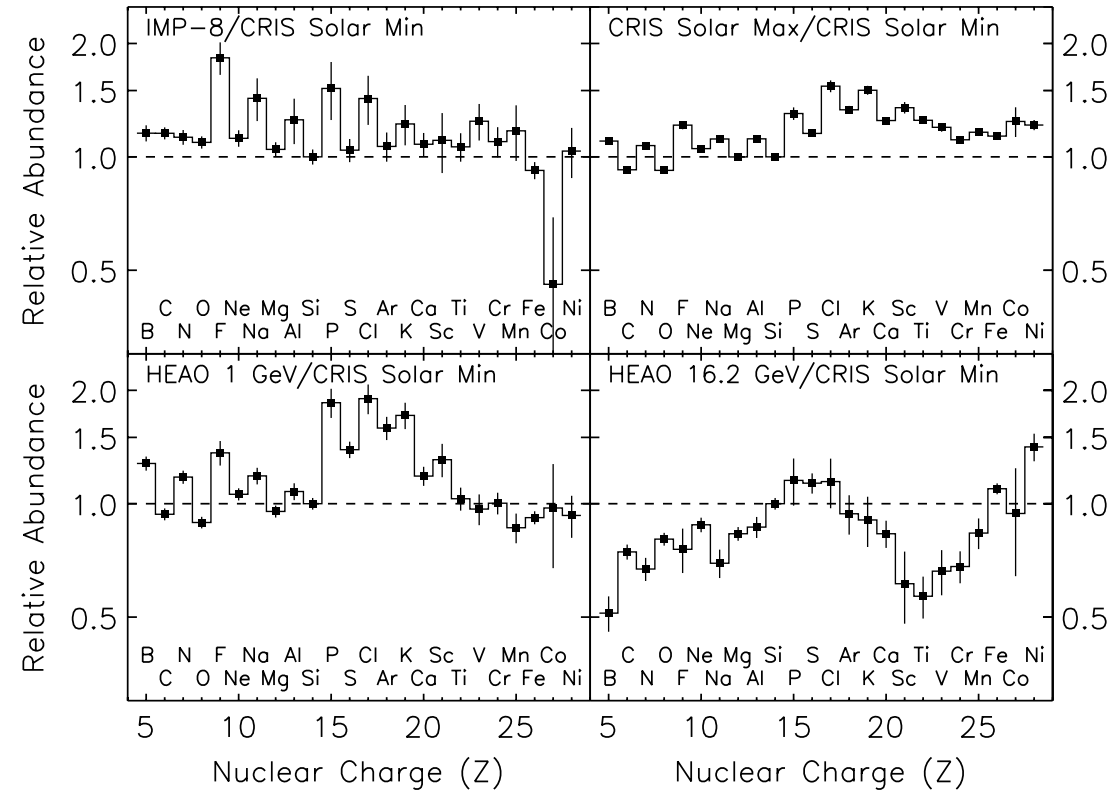

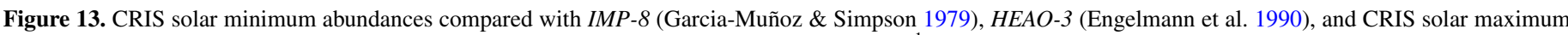
data. Abundances are normalized to silicon, with CRIS abundances given at $160 \mathrm{MeV} \mathrm{nucleon}^{-1}$. 


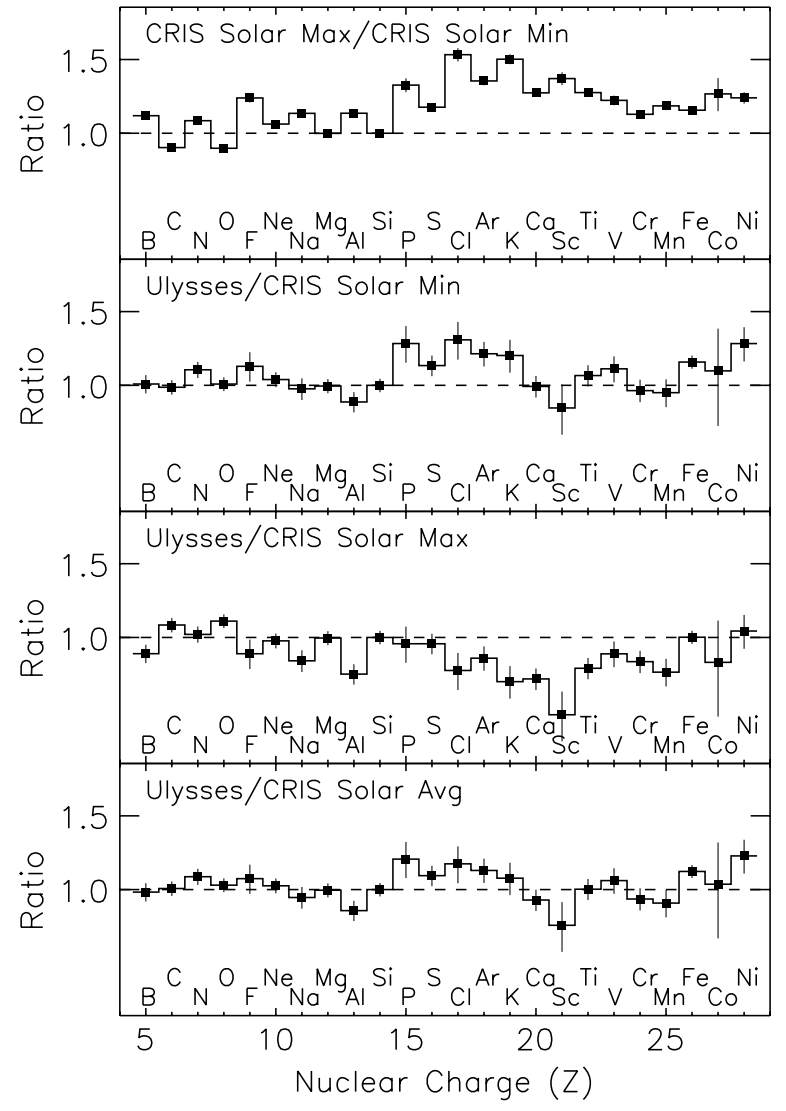

Figure 14. Ulysses abundances (DuVernois \& Thayer 1996) compared with CRIS solar minimum, CRIS solar maximum, and CRIS "solar average" (the arithmetic mean of the CRIS solar minimum and maximum data) normalized to Si. Also shown for reference are the abundance ratios for CRIS solar maximum/ minimum.

16.2 $\mathrm{GeV}_{\text {nucleon }}{ }^{-1}$. At $1 \mathrm{GeV}$ nucleon $^{-1}$, the $H E A O-3$ data show greater abundances for $\mathrm{B}, \mathrm{N}, \mathrm{F}$, and $\mathrm{Na}$, as well as for $15 \leqslant Z \leqslant 21$. This is not surprising since each of these elements have substantial secondary components, and the HEAO-3 composition at $1 \mathrm{GeV}$ nucleon ${ }^{-1}$ samples considerably longer path lengths in the Galaxy than the CRIS data at $160 \mathrm{MeV}$ nucleon $^{-1}$. Additionally, the HEAO-3 $\left(1 \mathrm{GeV}\right.$ nucleon $\left.^{-1}\right) / \mathrm{CRIS}$ solar minimum ratios are qualitatively similar to the CRIS solar maximum/minimum ratios. We expect this since CRIS samples particles that come from a higher energy distribution during solar maximum than it does at solar minimum.

At $16.2 \mathrm{GeV}$ nucleon $^{-1}$, we instead see a lower abundance of secondaries in the $H E A O-3$ composition. Relative to a primary element, secondaries are less abundant at higher energies than at lower energies. This was mentioned earlier, in reference to Figure 12: cosmic rays at high energies spend less time in the Galaxy before escaping, and therefore produce fewer secondaries.

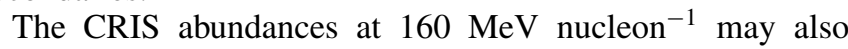
be compared with Ulysses abundances (DuVernois \& Thayer 1996), as seen in Figure 14. Since the Ulysses data were taken during the transition from a period of solar maximum to minimum, the data are compared with the CRIS solar minimum, solar maximum, and "solar average" abundances. "Solar average" abundances are simply the unweighted arithmetic mean of the CRIS solar minimum and maximum data. Also provided are the CRIS solar maximum/minimum ratios from Figure 13 as a reference for the three comparisons. As is seen, the Ulysses data are not in complete agreement with data from either CRIS solar minimum or maximum. The Ulysses results are in rather good agreement with the CRIS "solar average"; the only serious exceptions are the $>2 \sigma$ differences for aluminum and iron. Currently, we have no explanation for these discrepancies between the Ulysses and CRIS abundances, though Wiedenbeck et al. (2001) have previously noted a similar discrepancy for the sub-Fe/Fe ratio. We conclude that the Ulysses data may be seen as representing a "solar average" period.

\section{SUMMARY}

We have reported CRIS measurements of elemental spectra and abundances for GCRs from boron to nickel during the 19971998 solar minimum period and during the solar maximum period from 2001 to 2003. The large acceptance and excellent charge resolution of the instrument enable a high statistical significance for even the rare elements. The current data are generally consistent with previous measurements, allowing for differences in the average solar modulation level during the times the measurements were made. At energies below those measured in HEAO-3 (Engelmann et al. 1990), the CRIS data are much more precise than any previously available. Neutron monitor levels during the 1997-1998 period were consistently high and relatively constant, with none of the large decreases that affected the IMP-8 1974-1976 spectra. The CRIS solar minimum data thus represent a clean snapshot of the GCRs when the heliosphere is in its most quiescent state.

The ACE spacecraft and the CRIS instrument continue to function well and record GCR spectra and intensities for the current period of solar minimum. The CRIS elemental data are now available for the full solar cycle and are released to the space physics community on a regular basis. ${ }^{9}$ The measurements reported here serve as a high-precision baseline for continued studies of GCR composition, solar modulation over the solar cycle, space radiation hazards, and other applications.

This work is supported by NASA at the California Institute of Technology (under grant NNX08AI11G), the Jet Propulsion Laboratory, the Goddard Space Flight Center, and Washington University in St. Louis. We also acknowledge the use of the Climax neutron monitor data provided to the community by the University of New Hampshire under the National Science Foundation Grant ATM-0339527.

\section{APPENDIX A}

\section{INTENSITY CALCULATION}

The absolute intensity of particles measured by CRIS is a ratio of the number of counts detected to the product of various detector response factors, including the geometrical acceptance, exposure time, efficiencies, and the energy interval over which the detector is sensitive. The intensity at the top of the instrument is given by

$$
\text { Intensity }=\frac{N}{A \cdot \Omega \cdot \Delta \varepsilon \cdot \epsilon_{\mathrm{spall}} \cdot \epsilon_{\mathrm{SOFT}} \cdot t_{\text {live }}},
$$

where $N$ counts are observed in $t_{\text {live }}$ seconds of active exposure in an energy interval of $\Delta \varepsilon \mathrm{MeV}$ nucleon $^{-1}$ with geometrical

\footnotetext{
9 The CRIS elemental data are available from the $A C E$ Science Center archives at http://www.srl.caltech.edu/ACE/ASC/level2/ lvl2DATA_CRIS.html
} 


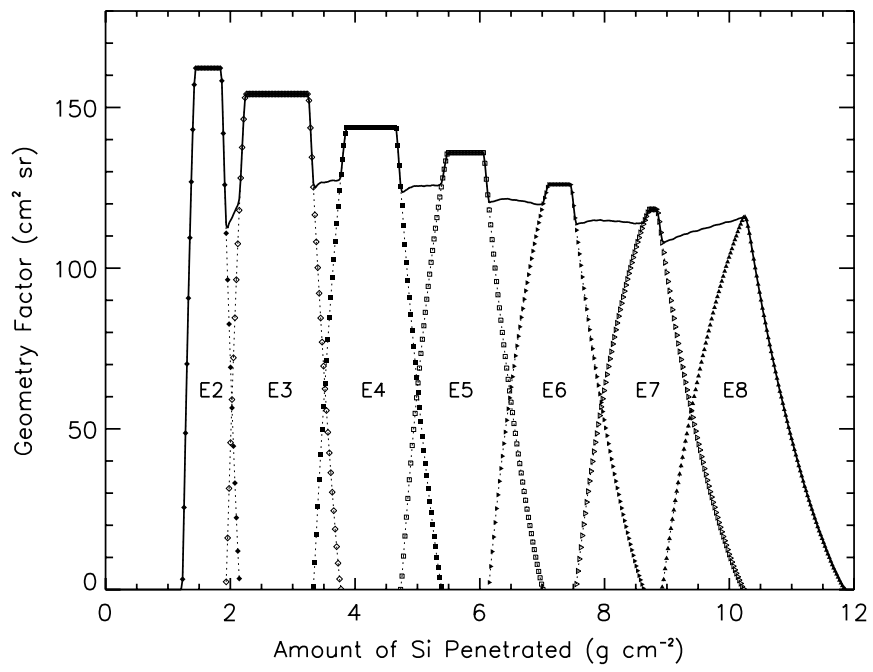

Figure 15. CRIS geometry factor vs. penetrated range of Si. The dotted lines with symbols show contributions to the geometry factor from particles stopping in individual detectors. The solid black line depicts the total acceptance of the instrument. The calculation uses the same geometrical cuts as were applied in the data analysis. Drops in the total acceptance between detectors come from the $160 \mu \mathrm{m}$ detector face cuts applied to avoid "dead layer" effects.

acceptance $A \Omega$. The remaining factors are efficiency corrections for the SOFT hodoscope, $\epsilon_{\mathrm{SOFT}}$, and for nuclear interactions occurring within the instrument, $\epsilon_{\text {spall }}$.

The CRIS intensities are most naturally determined in seven bins corresponding to particles stopping in detectors E2 through E8. The corresponding energy intervals for these bins slightly overlap due to the distribution of trajectory angles; however, the intensities determined in each bin are independent.

\section{A.1. Geometry Factor and Energies}

The geometrical acceptance, shown in Figure 15, was calculated using Monte Carlo techniques with the same cuts as were applied to the data analysis. The dotted lines show the contribution from individual detectors while the solid line gives the sum as a function of penetrated range in the silicon detectors. The factor $A \Omega \Delta \varepsilon$ in Equation (A1) is the differential geometry factor per unit energy, integrated over the energies of the particles stopping in a given detector, that is,

$$
A \Omega \Delta \varepsilon=\int\left(\frac{d(A \Omega)}{d \varepsilon}\right) d \varepsilon .
$$

The calculation uses $\sim 5000$ tracks per range bin, giving a statistical uncertainty $(1 / \sqrt{N})$ of $1.4 \%$. A second independent simulation gave agreement within $2 \%$ over all stopping particle ranges. We assign a $2 \%$ uncertainty to the $A \Omega \Delta \varepsilon$ product.

For particles stopping in one of the seven detectors, there is an associated "central energy" at which to plot the respective intensity. These energies are determined from the penetrated ranges at the endpoints of the individual geometry factors using the range-energy relation. Energy endpoints are determined for each isotope observed in the cosmic rays and weighted by the relative isotopic contributions. For boron $(Z=5)$ and heavier nuclei, even relatively large $(10 \%)$ uncertainties in the isotope fractions have a negligible effect on the width of the calculated energy interval defined by the endpoints. The central energy is taken as the arithmetic mean of the endpoints.

\section{A.2. Spallation Correction}

A spallation survival correction, $\epsilon_{\text {spall }}$, is made to account for incident nuclei eliminated from the analysis due to nuclear interactions occurring within the instrument. Energy independent cross sections for mass-changing interactions were calculated using a formula obtained by Westfall et al. (1979). Given these cross sections, the interaction length in silicon is $38.7 \mathrm{~g} \mathrm{~cm}^{-2}$ for ${ }^{12} \mathrm{C}$, and $20.8 \mathrm{~g} \mathrm{~cm}^{-2}$ for ${ }^{56} \mathrm{Fe}$. In scintillator, the interaction length is $21.8 \mathrm{~g} \mathrm{~cm}^{-2}$ for ${ }^{12} \mathrm{C}$ and $9.1 \mathrm{~g} \mathrm{~cm}^{-2}$ for ${ }^{56} \mathrm{Fe}$. For an aluminum target, the interaction lengths are similar to those for a silicon target, with $38.0 \mathrm{~g} \mathrm{~cm}^{-2}$ for ${ }^{12} \mathrm{C}$ and $20.3 \mathrm{~g} \mathrm{~cm}^{-2}$ for ${ }^{56} \mathrm{Fe}$.

When calculating the survival probability of an incident particle, the total amount of material traversed must be known. The thickness of the SOFT hodoscope and overlying material (the instrument cover and thermal blankets) amounts to $0.442 \mathrm{~g} \mathrm{~cm}^{-2}$ (Stone et al. 1998). For the purposes of calculating the correction factor, the material above the hodoscope is approximated to be made of aluminum and scintillator material. Together with the inferred depth of the particle in the silicon detectors and the angle of incidence, the total amount of material traversed may be determined.

The probability of survival without interaction in the instrument for a nucleus of charge $Z$ and energy such that the particle would stop at the middle depth of silicon detector $D$ $(D=2,3, \ldots, 8)$ is given by

$$
\begin{aligned}
& \epsilon_{\text {spall }}(Z, D)=\sum_{i=\text { isotopes }} w\left(Z, M_{i}\right) \\
& \quad \times \exp \left(-\frac{t_{\mathrm{Al}}}{X_{\mathrm{Al}}\left(M_{i}\right)}-\frac{t_{\mathrm{scint}}}{X_{\mathrm{scint}}\left(M_{i}\right)}-\frac{t_{\mathrm{Si}}(D)}{X_{\mathrm{Si}}\left(M_{i}\right)}\right) .
\end{aligned}
$$

where $t_{\mathrm{Al}}$ is the thickness of aluminum, $t_{\text {scint }}$ is the thickness of scintillator material, $t_{\mathrm{Si}}(D)$ is the amount of silicon traversed in stopping in detector $D$, and $X_{\mathrm{Al}}\left(M_{i}\right), X_{\mathrm{scint}}\left(M_{i}\right)$, and $X_{\mathrm{Si}}\left(M_{i}\right)$ are the interaction lengths in aluminum, scintillator, and silicon, respectively. The exponential is weighted by $w\left(Z, M_{i}\right)$, the isotopic fractions of element $Z$ observed in CRIS, to form an elemental average. The total thickness of each material is given by the actual thickness divided by the cosine of an average angle.

We assume that every interacting nucleus is eliminated based on charge and mass consistency criteria. The survival factors range from $96 \%$ for boron stopping in detector 2 to $60 \%$ for nickel stopping in detector 8 . They are plotted as a function of nuclear charge for all detectors in Figure 16. Assigning a 10\% uncertainty in the spallation cross sections, which is implied by the uncertainties in the parameters defined by Westfall et al. (1979), yields < $1 \%$ uncertainty in the spallation survival factor for all nuclei in the shallowest ranges, and $~ 3 \%-5 \%$ uncertainty in the factor for the deepest ranges.

\section{A.3. SOFT Efficiency}

The SOFT efficiency was calculated by selecting groups of events based solely on the stack detector pulse height data and observing the fraction that have valid trajectories in SOFT. These events are restricted to incident angles of $\theta<30^{\circ}$ since this is the part of the geometrical acceptance over which some of the major elements may be resolved without requiring the trajectory information. The result is plotted in Figure 17, parameterized by the energy loss in $\mathrm{Si}$ at the top of the instrument. Only 


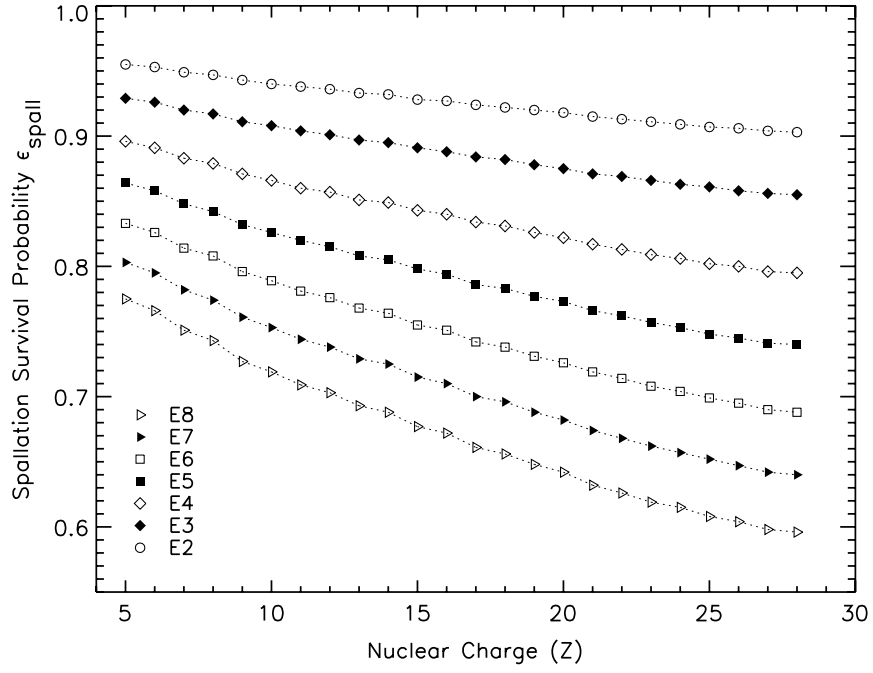

Figure 16. Spallation correction factors as a function of nuclear charge for particles stopping in each of the CRIS stack detectors.

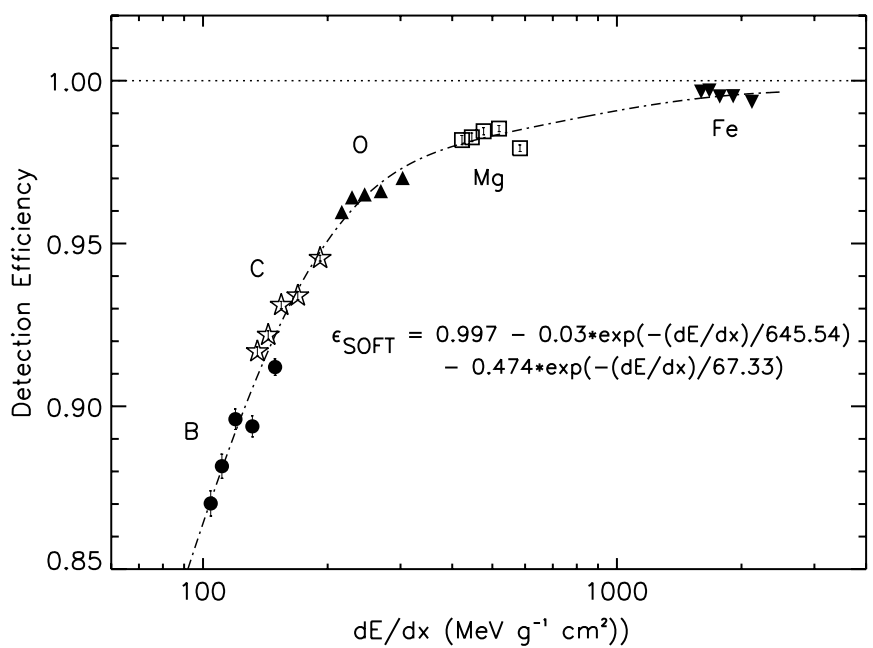

Figure 17. SOFT detection efficiency parameterized by the energy loss at the top of the instrument. The five points for each selected element correspond to, from right to left, particles stopping in detectors E4 through E8. The dashed line represents a fit to the measured points, given by the above equation. elements that could be cleanly separated in detectors E4 through E8 without using the hodoscope data were included. The fit curve allows interpolation for other species and detectors. The efficiency is above $87 \%$ for all elements treated in this paper, and is above $95 \%$ and relatively flat for elements heavier than carbon.

\section{A.4. Exposure Time}

Data used in the solar minimum spectra were recorded over a period of 236 days between 1997 August 28 and 1998 April 19. In this period, a total of 17 days $(7.2 \%)$ were removed for spacecraft activities, such as halo orbit insertion maneuvers, and for two solar energetic particle events on 1997 November 4 and 6 . The fractional live time for $Z>2$ particles is assessed by onboard timers and averaged over $80 \%$ (see Figure 18). The dead time is due primarily to instrument activities such as pulser calibrations, leakage current balancing, etc. (Stone et al. 1998). The threshold on a noisy guard ring channel was raised 1998 January 20 , eliminating a source of variation in the fractional live time.

Solar maximum data came from an 854 day period between 2001 May 1 and 2003 September 1. A total of 121 days (14.2\%) during this period were removed from the data set, nearly all for solar energetic particle events. Most solar energetic particle events do not cause any significant background in CRIS, but high intensities of low-energy particles increase the SOFT trigger rates, creating significant instrument dead time. In a few cases, the rates were high enough to trip current limits that turn off the SOFT high voltage supplies. These limits protect the microchannel plates used in the SOFT hodoscope; the supplies are turned back on via a command from the ground once particle intensities return to normal levels.

\section{APPENDIX B}

\section{CRIS ELEMENTAL SPECTRA}

This appendix provides the user with the observed elemental spectra for solar minimum (Table B1) and maximum (Table B2). These data were used to create Figures 5 and 6 in
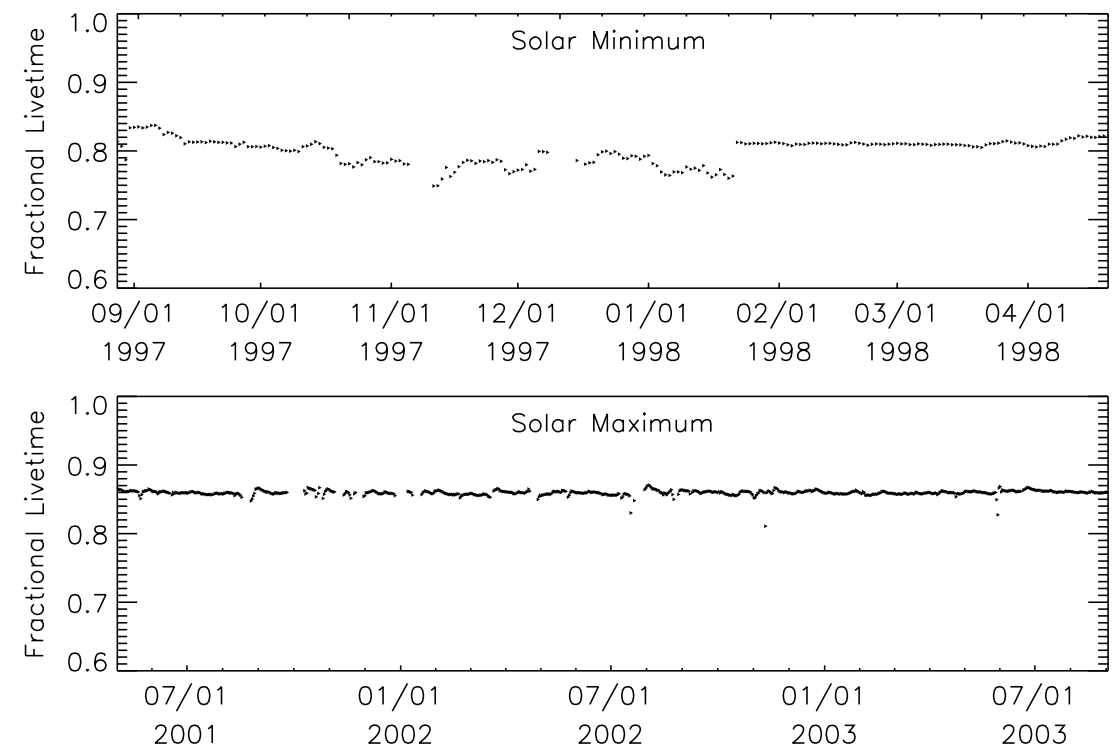

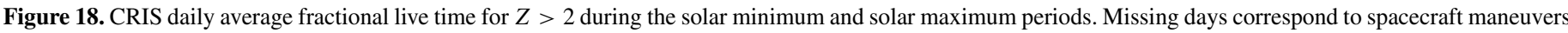
and solar energetic particle events. Variations before 1998 January 20 were due to a noisy guard ring. 
Table B1

CRIS Solar Minimum Spectra

\begin{tabular}{|c|c|c|c|c|c|c|c|}
\hline \multirow{2}{*}{$\begin{array}{c}\text { Element } \\
\mathrm{B}\end{array}$} & \multicolumn{7}{|c|}{$\begin{array}{c}\text { Energies }\left(\mathrm{MeV} \text { nucleon }^{-1}\right) \\
\text { Intensities }\left(10^{-9}\left(\mathrm{~cm}^{2} \mathrm{~s} \text { sr MeV nucleon }{ }^{-1}\right)^{-1}\right)\end{array}$} \\
\hline & 59.5 & 79.7 & 102.0 & 121.2 & 138.5 & 154.2 & 169.0 \\
\hline & $108.8 \pm 3.7$ & $131.2 \pm 4.2$ & $155.5 \pm 5.2$ & $166.9 \pm 6.0$ & $184.2 \pm 7.0$ & $192.5 \pm 7.9$ & $195.2 \pm 8.5$ \\
\hline \multirow[t]{2}{*}{$\mathrm{C}$} & 68.2 & 91.5 & 117.2 & 139.4 & 159.4 & 177.7 & 194.8 \\
\hline & $517.6 \pm 15.4$ & $616.0 \pm 18.6$ & $714.9 \pm 22.6$ & $759.8 \pm 25.5$ & $797.7 \pm 28.6$ & $816.8 \pm 31.4$ & $795.1 \pm 32.8$ \\
\hline \multirow[t]{2}{*}{$\mathrm{N}$} & 73.1 & 98.1 & 125.8 & 149.7 & 171.3 & 191.0 & 209.5 \\
\hline & $126.8 \pm 4.1$ & $143.9 \pm 4.5$ & $170.3 \pm 5.7$ & $182.7 \pm 6.5$ & $191.5 \pm 7.5$ & $196.1 \pm 8.2$ & $189.2 \pm 8.5$ \\
\hline \multirow[t]{2}{*}{$\mathrm{O}$} & 80.3 & 107.9 & 138.4 & 164.9 & 188.8 & 210.6 & 231.1 \\
\hline & $573.6 \pm 17.0$ & $664.9 \pm 20.2$ & $735.9 \pm 23.5$ & $770.3 \pm 26.6$ & $775.5 \pm 28.7$ & $799.9 \pm 32.3$ & $756.7 \pm 33.4$ \\
\hline \multirow[t]{2}{*}{$\mathrm{F}$} & 83.3 & 112.0 & 143.8 & 171.4 & 196.3 & 219.1 & 240.5 \\
\hline & $7.4 \pm 0.5$ & $8.9 \pm 0.5$ & $10.6 \pm 0.6$ & $11.9 \pm 0.7$ & $10.5 \pm 0.7$ & $11.8 \pm 0.8$ & $11.4 \pm 0.8$ \\
\hline \multirow[t]{2}{*}{$\mathrm{Ne}$} & 89.3 & 120.1 & 154.3 & 184.0 & 210.9 & 235.6 & 258.7 \\
\hline & $82.4 \pm 2.7$ & $96.1 \pm 3.1$ & $107.7 \pm 3.8$ & $110.3 \pm 4.2$ & $110.6 \pm 4.6$ & $113.5 \pm 5.2$ & $112.2 \pm 5.5$ \\
\hline \multirow[t]{2}{*}{$\mathrm{Na}$} & 93.8 & 126.3 & 162.4 & 193.8 & 222.2 & 248.2 & 272.8 \\
\hline & $16.2 \pm 0.7$ & $18.8 \pm 0.8$ & $20.9 \pm 0.9$ & $20.2 \pm 1.0$ & $21.7 \pm 1.1$ & $22.9 \pm 1.3$ & $21.1 \pm 1.3$ \\
\hline \multirow[t]{2}{*}{$\mathrm{Mg}$} & 100.0 & 134.7 & 173.3 & 207.0 & 237.5 & 265.5 & 292.0 \\
\hline & $120.6 \pm 3.8$ & $136.4 \pm 4.3$ & $149.9 \pm 5.2$ & $156.7 \pm 5.9$ & $153.6 \pm 6.4$ & $153.3 \pm 7.0$ & $146.8 \pm 7.3$ \\
\hline \multirow[t]{2}{*}{$\mathrm{Al}$} & 103.6 & 139.7 & 179.8 & 214.9 & 246.6 & 275.8 & 303.4 \\
\hline & $17.6 \pm 0.8$ & $20.4 \pm 0.8$ & $22.7 \pm 1.0$ & $22.8 \pm 1.1$ & $23.2 \pm 1.2$ & $23.5 \pm 1.3$ & $21.5 \pm 1.3$ \\
\hline \multirow[t]{2}{*}{$\mathrm{Si}$} & 109.9 & 148.2 & 191.0 & 228.4 & 262.3 & 293.6 & 323.1 \\
\hline & $91.9 \pm 3.0$ & $104.6 \pm 3.4$ & $110.9 \pm 3.9$ & $111.9 \pm 4.4$ & $112.9 \pm 4.8$ & $110.4 \pm 5.3$ & $103.4 \pm 5.4$ \\
\hline \multirow[t]{2}{*}{$\mathrm{P}$} & 112.5 & 151.9 & 195.9 & 234.3 & 269.2 & 301.4 & 331.8 \\
\hline & $2.4 \pm 0.2$ & $2.7 \pm 0.2$ & $3.1 \pm 0.2$ & $3.1 \pm 0.3$ & $3.5 \pm 0.3$ & $2.9 \pm 0.3$ & $3.5 \pm 0.4$ \\
\hline \multirow[t]{2}{*}{ S } & 118.0 & 159.4 & 205.6 & 246.1 & 283.0 & 317.0 & 349.1 \\
\hline & $14.2 \pm 0.6$ & $16.7 \pm 0.7$ & $18.5 \pm 0.8$ & $18.8 \pm 0.9$ & $18.5 \pm 1.0$ & $18.2 \pm 1.1$ & $17.9 \pm 1.2$ \\
\hline \multirow[t]{2}{*}{$\mathrm{Cl}$} & 119.8 & 161.9 & 209.0 & 250.2 & 287.8 & 322.4 & 355.2 \\
\hline & $2.5 \pm 0.2$ & $2.5 \pm 0.2$ & $3.1 \pm 0.2$ & $3.2 \pm 0.3$ & $3.6 \pm 0.3$ & $3.6 \pm 0.3$ & $3.2 \pm 0.3$ \\
\hline \multirow[t]{2}{*}{$\mathrm{Ar}$} & 124.9 & 168.9 & 218.2 & 261.5 & 300.8 & 337.3 & 371.7 \\
\hline & $5.6 \pm 0.3$ & $6.5 \pm 0.3$ & $6.8 \pm 0.4$ & $7.4 \pm 0.5$ & $8.0 \pm 0.5$ & $7.5 \pm 0.6$ & $7.1 \pm 0.6$ \\
\hline \multirow[t]{2}{*}{ K } & 127.7 & 172.8 & 223.3 & 267.7 & 308.1 & 345.5 & 380.9 \\
\hline & $4.0 \pm 0.3$ & $4.5 \pm 0.2$ & $5.3 \pm 0.3$ & $5.4 \pm 0.4$ & $5.5 \pm 0.4$ & $5.9 \pm 0.5$ & $5.2 \pm 0.5$ \\
\hline \multirow[t]{2}{*}{$\mathrm{Ca}$} & 131.5 & 178.2 & 230.4 & 276.3 & 318.2 & 356.9 & 393.7 \\
\hline & $11.7 \pm 0.5$ & $14.6 \pm 0.6$ & $14.9 \pm 0.7$ & $15.8 \pm 0.8$ & $15.4 \pm 0.9$ & $16.4 \pm 1.0$ & $14.9 \pm 1.0$ \\
\hline \multirow[t]{2}{*}{$\mathrm{Sc}$} & 133.3 & 180.6 & 233.7 & 280.3 & 322.8 & 362.3 & 399.6 \\
\hline & $2.6 \pm 0.2$ & $2.8 \pm 0.2$ & $3.0 \pm 0.2$ & $3.4 \pm 0.3$ & $3.2 \pm 0.3$ & $2.9 \pm 0.3$ & $3.0 \pm 0.3$ \\
\hline \multirow[t]{2}{*}{$\mathrm{Ti}$} & 136.8 & 185.6 & 240.2 & 288.3 & 332.2 & 372.9 & 411.5 \\
\hline & $10.0 \pm 0.5$ & $11.2 \pm 0.5$ & $12.5 \pm 0.6$ & $12.2 \pm 0.7$ & $12.0 \pm 0.7$ & $12.0 \pm 0.8$ & $11.4 \pm 0.9$ \\
\hline \multirow[t]{2}{*}{ V } & 139.7 & 189.5 & 245.5 & 294.7 & 339.7 & 381.5 & 421.1 \\
\hline & $4.5 \pm 0.3$ & $5.5 \pm 0.3$ & $5.3 \pm 0.3$ & $5.3 \pm 0.4$ & $5.7 \pm 0.4$ & $5.1 \pm 0.4$ & $5.4 \pm 0.5$ \\
\hline \multirow[t]{2}{*}{$\mathrm{Cr}$} & 143.6 & 195.0 & 252.7 & 303.6 & 350.1 & 393.3 & 434.4 \\
\hline & $10.2 \pm 0.5$ & $11.1 \pm 0.5$ & $11.4 \pm 0.6$ & $11.4 \pm 0.6$ & $11.3 \pm 0.7$ & $10.8 \pm 0.8$ & $10.1 \pm 0.8$ \\
\hline $\mathrm{Mn}$ & 146.5 & 199.1 & 258.1 & 310.2 & 357.9 & 402.2 & 444.2 \\
\hline & $6.5 \pm 0.4$ & $6.8 \pm 0.3$ & $7.3 \pm 0.4$ & $7.1 \pm 0.4$ & $6.8 \pm 0.5$ & $6.3 \pm 0.5$ & $5.2 \pm 0.5$ \\
\hline $\mathrm{Fe}$ & 150.2 & 204.2 & 265.0 & 318.6 & 367.8 & 413.4 & 456.9 \\
\hline & $68.7 \pm 2.3$ & $73.8 \pm 2.6$ & $73.9 \pm 2.9$ & $69.3 \pm 3.1$ & $68.0 \pm 3.5$ & $62.5 \pm 3.6$ & $59.5 \pm 3.8$ \\
\hline Co & 153.3 & 208.5 & 270.7 & 325.6 & 376.0 & 422.8 & 467.4 \\
\hline & $0.54 \pm 0.08$ & $0.29 \pm 0.05$ & $0.44 \pm 0.07$ & $0.32 \pm 0.07$ & $0.47 \pm 0.09$ & $0.35 \pm 0.09$ & $0.34 \pm 0.09$ \\
\hline $\mathrm{Ni}$ & 158.7 & 216.1 & 280.8 & 338.0 & 390.5 & 439.4 & 486.0 \\
\hline & $3.0 \pm 0.2$ & $3.2 \pm 0.2$ & $3.5 \pm 0.2$ & $3.5 \pm 0.3$ & $3.2 \pm 0.3$ & $2.8 \pm 0.3$ & $3.0 \pm 0.3$ \\
\hline
\end{tabular}

Note. The systematic and statistical uncertainties are combined in quadrature.

Section 4. Details of the intensity calculation and associated correction factors are provided in Appendix A.

For the convenience of the user, the CRIS measurements have also been linearly interpolated between adjacent data points in $\log$ (Intensity) versus $\log$ (Energy/nucleon) to a common energy grid, given in Tables B3 and B4. In both tables, we have included slightly extrapolated data points for several of the elements at the high and/or low energies. Uncertainties in the interpolated points are calculated by combining in quadrature the overall systematic uncertainty with a statistical contribution, which is a simple propagation of errors from the statistical uncertainties of the values on either side of the interpolated point.

\section{APPENDIX C}

\section{COSMIC-RAY TRANSPORT MODELING}

Modeling of cosmic-ray transport from galactic sources to Earth was carried out in two parts, using simple models of interstellar propagation and solar modulation. This appendix summarizes the calculations that were performed.

\section{C.1. Interstellar Propagation}

Energy spectra observed near Earth were interpreted using a steady-state leaky box propagation model based on the 
Table B2

CRIS Solar Maximum Spectra

\begin{tabular}{|c|c|c|c|c|c|c|c|}
\hline \multirow{2}{*}{$\begin{array}{c}\text { Element } \\
\mathrm{B}\end{array}$} & \multicolumn{7}{|c|}{$\begin{array}{c}\text { Energies }\left(\mathrm{MeV} \text { nucleon }^{-1}\right) \\
\text { Intensities }\left(10^{-9}\left(\mathrm{~cm}^{2} \mathrm{~s} \text { sr MeV nucleon }{ }^{-1}\right)^{-1}\right)\end{array}$} \\
\hline & 59.5 & 79.7 & 102.0 & 121.2 & 138.5 & 154.2 & 169.0 \\
\hline & $29.3 \pm 1.0$ & $36.1 \pm 1.2$ & $43.2 \pm 1.5$ & $49.2 \pm 1.7$ & $53.3 \pm 2.0$ & $57.7 \pm 2.3$ & $58.1 \pm 2.5$ \\
\hline \multirow[t]{2}{*}{$\mathrm{C}$} & 68.2 & 91.5 & 117.2 & 139.4 & 159.4 & 177.7 & 194.8 \\
\hline & $108.9 \pm 3.3$ & $134.1 \pm 4.1$ & $163.0 \pm 5.2$ & $182.4 \pm 6.1$ & $201.3 \pm 7.2$ & $208.1 \pm 8.0$ & $215.8 \pm 8.9$ \\
\hline \multirow{2}{*}{$\mathrm{N}$} & 73.1 & 98.1 & 125.8 & 149.7 & 171.3 & 191.0 & 209.5 \\
\hline & $31.0 \pm 1.0$ & $37.4 \pm 1.2$ & $46.3 \pm 1.6$ & $51.9 \pm 1.9$ & $56.3 \pm 2.2$ & $60.0 \pm 2.5$ & $59.8 \pm 2.7$ \\
\hline \multirow[t]{2}{*}{$\mathrm{O}$} & 80.3 & 107.9 & 138.4 & 164.9 & 188.8 & 210.6 & 231.1 \\
\hline & $121.7 \pm 3.6$ & $147.2 \pm 4.5$ & $177.7 \pm 5.7$ & $192.1 \pm 6.6$ & $207.0 \pm 7.7$ & $217.5 \pm 8.8$ & $220.2 \pm 9.7$ \\
\hline \multirow[t]{2}{*}{$\mathrm{F}$} & 83.3 & 112.0 & 143.8 & 171.4 & 196.3 & 219.1 & 240.5 \\
\hline & $2.3 \pm 0.1$ & $3.0 \pm 0.1$ & $3.3 \pm 0.2$ & $3.8 \pm 0.2$ & $3.9 \pm 0.2$ & $4.1 \pm 0.3$ & $4.4 \pm 0.3$ \\
\hline \multirow[t]{2}{*}{$\mathrm{Ne}$} & 89.3 & 120.1 & 154.3 & 184.0 & 210.9 & 235.6 & 258.7 \\
\hline & $20.6 \pm 0.7$ & $25.5 \pm 0.8$ & $29.8 \pm 1.0$ & $32.3 \pm 1.2$ & $36.2 \pm 1.5$ & $37.3 \pm 1.7$ & $36.1 \pm 1.8$ \\
\hline \multirow[t]{2}{*}{$\mathrm{Na}$} & 93.8 & 126.3 & 162.4 & 193.8 & 222.2 & 248.2 & 272.8 \\
\hline & $4.5 \pm 0.2$ & $5.4 \pm 0.2$ & $6.3 \pm 0.3$ & $6.6 \pm 0.3$ & $7.2 \pm 0.4$ & $7.7 \pm 0.4$ & $7.8 \pm 0.5$ \\
\hline \multirow[t]{2}{*}{$\mathrm{Mg}$} & 100.0 & 134.7 & 173.3 & 207.0 & 237.5 & 265.5 & 292.0 \\
\hline & $29.0 \pm 0.9$ & $35.6 \pm 1.1$ & $41.7 \pm 1.4$ & $45.1 \pm 1.7$ & $47.6 \pm 2.0$ & $50.4 \pm 2.3$ & $49.3 \pm 2.4$ \\
\hline \multirow[t]{2}{*}{$\mathrm{Al}$} & 103.6 & 139.7 & 179.8 & 214.9 & 246.6 & 275.8 & 303.4 \\
\hline & $4.7 \pm 0.2$ & $5.9 \pm 0.2$ & $7.0 \pm 0.3$ & $7.8 \pm 0.4$ & $8.3 \pm 0.4$ & $7.9 \pm 0.4$ & $8.4 \pm 0.5$ \\
\hline \multirow[t]{2}{*}{$\mathrm{Si}$} & 109.9 & 148.2 & 191.0 & 228.4 & 262.3 & 293.6 & 323.1 \\
\hline & $23.3 \pm 0.8$ & $27.8 \pm 0.9$ & $31.9 \pm 1.1$ & $33.9 \pm 1.3$ & $36.2 \pm 1.5$ & $38.5 \pm 1.8$ & $37.6 \pm 2.0$ \\
\hline \multirow[t]{2}{*}{$\mathrm{P}$} & 112.5 & 151.9 & 195.9 & 234.3 & 269.2 & 301.4 & 331.8 \\
\hline & $0.7 \pm 0.1$ & $1.1 \pm 0.1$ & $1.1 \pm 0.1$ & $1.2 \pm 0.1$ & $1.4 \pm 0.1$ & $1.6 \pm 0.1$ & $1.6 \pm 0.1$ \\
\hline \multirow[t]{2}{*}{ S } & 118.0 & 159.4 & 205.6 & 246.1 & 283.0 & 317.0 & 349.1 \\
\hline & $4.3 \pm 0.2$ & $5.1 \pm 0.2$ & $6.2 \pm 0.3$ & $6.9 \pm 0.3$ & $6.8 \pm 0.3$ & $7.5 \pm 0.4$ & $7.5 \pm 0.5$ \\
\hline \multirow[t]{2}{*}{$\mathrm{Cl}$} & 119.8 & 161.9 & 209.0 & 250.2 & 287.8 & 322.4 & 355.2 \\
\hline & $0.9 \pm 0.1$ & $1.1 \pm 0.1$ & $1.4 \pm 0.1$ & $1.4 \pm 0.1$ & $1.5 \pm 0.1$ & $1.9 \pm 0.1$ & $1.7 \pm 0.1$ \\
\hline \multirow[t]{2}{*}{$\mathrm{Ar}$} & 124.9 & 168.9 & 218.2 & 261.5 & 300.8 & 337.3 & 371.7 \\
\hline & $1.9 \pm 0.1$ & $2.3 \pm 0.1$ & $2.8 \pm 0.1$ & $3.2 \pm 0.2$ & $3.2 \pm 0.2$ & $3.3 \pm 0.2$ & $3.1 \pm 0.2$ \\
\hline \multirow[t]{2}{*}{$\mathrm{K}$} & 127.7 & 172.8 & 223.3 & 267.7 & 308.1 & 345.5 & 380.9 \\
\hline & $1.6 \pm 0.1$ & $1.8 \pm 0.1$ & $2.2 \pm 0.1$ & $2.4 \pm 0.1$ & $2.5 \pm 0.2$ & $2.6 \pm 0.2$ & $2.7 \pm 0.2$ \\
\hline \multirow[t]{2}{*}{$\mathrm{Ca}$} & 131.5 & 178.2 & 230.4 & 276.3 & 318.2 & 356.9 & 393.7 \\
\hline & $3.9 \pm 0.2$ & $5.0 \pm 0.2$ & $5.4 \pm 0.2$ & $5.9 \pm 0.3$ & $6.3 \pm 0.3$ & $6.6 \pm 0.4$ & $6.6 \pm 0.4$ \\
\hline \multirow[t]{2}{*}{$\mathrm{Sc}$} & 133.3 & 180.6 & 233.7 & 280.3 & 322.8 & 362.3 & 399.6 \\
\hline & $0.9 \pm 0.1$ & $1.1 \pm 0.1$ & $1.2 \pm 0.1$ & $1.2 \pm 0.1$ & $1.4 \pm 0.1$ & $1.4 \pm 0.1$ & $1.4 \pm 0.1$ \\
\hline \multirow[t]{2}{*}{$\mathrm{Ti}$} & 136.8 & 185.6 & 240.2 & 288.3 & 332.2 & 372.9 & 411.5 \\
\hline & $3.3 \pm 0.2$ & $4.0 \pm 0.2$ & $4.3 \pm 0.2$ & $4.6 \pm 0.2$ & $4.6 \pm 0.3$ & $4.8 \pm 0.3$ & $4.6 \pm 0.3$ \\
\hline \multirow[t]{2}{*}{ V } & 139.7 & 189.5 & 245.5 & 294.7 & 339.7 & 381.5 & 421.1 \\
\hline & $1.4 \pm 0.1$ & $1.8 \pm 0.1$ & $2.0 \pm 0.1$ & $2.1 \pm 0.1$ & $2.2 \pm 0.1$ & $2.0 \pm 0.2$ & $2.3 \pm 0.2$ \\
\hline \multirow[t]{2}{*}{$\mathrm{Cr}$} & 143.6 & 195.0 & 252.7 & 303.6 & 350.1 & 393.3 & 434.4 \\
\hline & $2.9 \pm 0.1$ & $3.7 \pm 0.2$ & $3.9 \pm 0.2$ & $4.0 \pm 0.2$ & $4.4 \pm 0.3$ & $4.4 \pm 0.3$ & $4.1 \pm 0.3$ \\
\hline $\mathrm{Mn}$ & 146.5 & 199.1 & 258.1 & 310.2 & 357.9 & 402.2 & 444.2 \\
\hline & $2.0 \pm 0.1$ & $2.1 \pm 0.1$ & $2.6 \pm 0.1$ & $2.6 \pm 0.2$ & $2.8 \pm 0.2$ & $2.8 \pm 0.2$ & $2.6 \pm 0.2$ \\
\hline $\mathrm{Fe}$ & 150.2 & 204.2 & 265.0 & 318.6 & 367.8 & 413.4 & 456.9 \\
\hline & $21.0 \pm 0.7$ & $23.7 \pm 0.8$ & $26.3 \pm 1.0$ & $27.2 \pm 1.2$ & $28.6 \pm 1.4$ & $28.3 \pm 1.6$ & $27.9 \pm 1.8$ \\
\hline Co & 153.3 & 208.5 & 270.7 & 325.6 & 376.0 & 422.8 & 467.4 \\
\hline & $0.09 \pm 0.02$ & $0.14 \pm 0.02$ & $0.19 \pm 0.02$ & $0.17 \pm 0.03$ & $0.17 \pm 0.03$ & $0.20 \pm 0.04$ & $0.20 \pm 0.04$ \\
\hline $\mathrm{Ni}$ & 158.7 & 216.1 & 280.8 & 338.0 & 390.5 & 439.4 & 486.0 \\
\hline & $1.0 \pm 0.1$ & $1.2 \pm 0.1$ & $1.3 \pm 0.1$ & $1.4 \pm 0.1$ & $1.6 \pm 0.1$ & $1.5 \pm 0.1$ & $1.4 \pm 0.1$ \\
\hline
\end{tabular}

Note. The systematic and statistical uncertainties are combined in quadrature.

formalism of Meneguzzi et al. (1971):

$$
\begin{aligned}
q_{i} & +\sum_{j} \varphi_{j}\left(\frac{1}{\Lambda_{\text {spall }}^{j i}}+\frac{1}{\Lambda_{\text {decay }}^{j i}}+\frac{1}{\Lambda_{\delta Q}^{j i}}\right) \\
& =\varphi_{i}\left(\frac{1}{\Lambda_{\text {spall }}^{i}}+\frac{1}{\Lambda_{\text {decay }}^{i}}+\frac{1}{\Lambda_{\delta Q}^{i}}+\frac{1}{\Lambda_{\text {esc }}^{i}}\right)-\frac{d}{d \varepsilon}\left(w_{i} \varphi_{i}\right)
\end{aligned}
$$

Here, the $\varphi_{i}$ represents the equilibrium interstellar intensity as a function of energy per nucleon, $\varepsilon$, for a particular cosmic-ray ion species specified by its atomic number, $Z_{i}$, mass number, $M_{i}$, and ionic charge state, $Q_{i}$. For the elements and energies of interest for the present study, most nuclei are fully stripped $\left(Q_{i}=Z_{i}\right)$ and only a small fraction have one electron attached $\left(Q_{i}=Z_{i}-1\right)$. Only these two charge states are considered in the calculation.

The terms on the left-hand side of Equation $(\mathrm{C} 1)$ represent the production of ion species $i$ in the cosmic-ray source $\left(q_{i}\right)$ and by nuclear spallation, radioactive decay, or attachment or loss of an orbital electron, with corresponding interstellar mean free paths denoted by $\Lambda_{\text {spall }}^{j i}, \Lambda_{\text {decay }}^{j i}$, and $\Lambda_{\delta Q}^{j i}$, respectively. The summation is over all species $j$ that can produce species $i$ by means of such interactions. On the right are the corresponding three loss terms for species $i$, with mean free paths $\Lambda_{\text {spall }}^{i}, \Lambda_{\text {decay }}^{i}$, and $\Lambda_{\delta Q}^{i}$. 
Table B3

CRIS Solar Minimum Spectra Interpolated to a Common Energy Grid

\begin{tabular}{|c|c|c|c|c|c|c|c|}
\hline \multicolumn{8}{|c|}{$\begin{array}{c}\text { Energies }\left(\mathrm{MeV} \text { nucleon }^{-1}\right) \\
\text { Intensities }\left(10^{-9}\left(\mathrm{~cm}^{2} \mathrm{~s} \text { sr MeV nucleon }{ }^{-1}\right)^{-1}\right)\end{array}$} \\
\hline Element & 60 & 72 & 85 & 100 & 120 & 142 & 170 \\
\hline B & $109.4 \pm 3.6$ & $123.0 \pm 3.8$ & $137.2 \pm 4.3$ & $153.4 \pm 5.1$ & $166.2 \pm 5.9$ & $186.1 \pm 6.9$ & $195.4 \pm 8.4$ \\
\hline $\mathrm{C}$ & & $534.5 \pm 15.7$ & $589.7 \pm 17.7$ & $649.8 \pm 19.5$ & $720.9 \pm 22.7$ & $764.9 \pm 25.6$ & $809.0 \pm 30.8$ \\
\hline $\mathrm{N}$ & & $126.0 \pm 4.1$ & $135.3 \pm 4.2$ & $145.8 \pm 4.6$ & $165.0 \pm 5.4$ & $178.8 \pm 6.2$ & $191.0 \pm 7.4$ \\
\hline $\mathrm{O}$ & & & $590.2 \pm 17.3$ & $640.1 \pm 19.4$ & $694.4 \pm 21.0$ & $740.9 \pm 23.6$ & $771.4 \pm 26.5$ \\
\hline $\mathrm{F}$ & & & $7.5 \pm 0.4$ & $8.3 \pm 0.4$ & $9.4 \pm 0.4$ & $10.5 \pm 0.5$ & $11.8 \pm 0.7$ \\
\hline $\mathrm{Ne}$ & & & $80.3 \pm 2.8$ & $87.4 \pm 2.7$ & $96.1 \pm 3.1$ & $103.8 \pm 3.5$ & $109.1 \pm 4.0$ \\
\hline $\mathrm{Na}$ & & & & $16.7 \pm 0.7$ & $18.3 \pm 0.7$ & $19.8 \pm 0.7$ & $20.7 \pm 0.8$ \\
\hline $\mathrm{Mg}$ & & & & $120.6 \pm 3.8$ & $130.1 \pm 4.1$ & $139.1 \pm 4.4$ & $148.8 \pm 5.1$ \\
\hline $\mathrm{Al}$ & & & & $17.3 \pm 0.8$ & $19.0 \pm 0.7$ & $20.6 \pm 0.8$ & $22.1 \pm 0.9$ \\
\hline $\mathrm{Si}$ & & & & & $95.5 \pm 3.0$ & $102.7 \pm 3.3$ & $108.0 \pm 3.7$ \\
\hline $\mathrm{P}$ & & & & & $2.4 \pm 0.2$ & $2.6 \pm 0.2$ & $2.9 \pm 0.2$ \\
\hline $\mathrm{S}$ & & & & & $14.3 \pm 0.6$ & $15.7 \pm 0.6$ & $17.1 \pm 0.6$ \\
\hline $\mathrm{Cl}$ & & & & & $2.5 \pm 0.2$ & $2.5 \pm 0.1$ & $2.6 \pm 0.2$ \\
\hline $\mathrm{Ar}$ & & & & & $5.5 \pm 0.4$ & $6.0 \pm 0.3$ & $6.5 \pm 0.3$ \\
\hline $\mathrm{K}$ & & & & & & $4.1 \pm 0.2$ & $4.4 \pm 0.2$ \\
\hline $\mathrm{Ca}$ & & & & & & $12.4 \pm 0.5$ & $14.1 \pm 0.5$ \\
\hline $\mathrm{Sc}$ & & & & & & $2.7 \pm 0.2$ & $2.8 \pm 0.2$ \\
\hline $\mathrm{Ti}$ & & & & & & $10.2 \pm 0.5$ & $10.8 \pm 0.4$ \\
\hline $\mathrm{V}$ & & & & & & $4.5 \pm 0.3$ & $5.1 \pm 0.2$ \\
\hline $\mathrm{Cr}$ & & & & & & $10.2 \pm 0.5$ & $10.7 \pm 0.4$ \\
\hline $\mathrm{Mn}$ & & & & & & $6.5 \pm 0.4$ & $6.6 \pm 0.3$ \\
\hline $\mathrm{Fe}$ & & & & & & & $70.7 \pm 2.4$ \\
\hline Co & & & & & & & $0.43 \pm 0.05$ \\
\hline $\mathrm{Ni}$ & & & & & & & $3.1 \pm 0.2$ \\
\hline Element & 200 & 240 & 285 & 340 & 400 & 475 & \\
\hline $\mathrm{C}$ & $789.0 \pm 32.3$ & & & & & & \\
\hline $\mathrm{N}$ & $192.6 \pm 8.3$ & & & & & & \\
\hline $\mathrm{O}$ & $788.3 \pm 31.6$ & $739.8 \pm 32.4$ & & & & & \\
\hline $\mathrm{F}$ & $10.7 \pm 0.6$ & $11.4 \pm 0.8$ & & & & & \\
\hline $\mathrm{Ne}$ & $110.5 \pm 4.4$ & $113.2 \pm 5.0$ & & & & & \\
\hline $\mathrm{Na}$ & $20.5 \pm 0.9$ & $22.6 \pm 1.2$ & $20.3 \pm 1.1$ & & & & \\
\hline $\mathrm{Mg}$ & $155.3 \pm 5.8$ & $153.5 \pm 6.4$ & $148.4 \pm 7.2$ & & & & \\
\hline $\mathrm{Al}$ & $22.8 \pm 1.0$ & $23.1 \pm 1.1$ & $22.8 \pm 1.2$ & & & & \\
\hline $\mathrm{Si}$ & $111.1 \pm 3.9$ & $112.3 \pm 4.3$ & $111.1 \pm 5.2$ & & & & \\
\hline $\mathrm{P}$ & $3.1 \pm 0.2$ & $3.2 \pm 0.2$ & $3.2 \pm 0.3$ & $3.7 \pm 0.3$ & & & \\
\hline $\mathrm{S}$ & $18.3 \pm 0.8$ & $18.7 \pm 0.9$ & $18.5 \pm 1.0$ & $18.0 \pm 1.1$ & & & \\
\hline $\mathrm{Cl}$ & $3.0 \pm 0.2$ & $3.1 \pm 0.2$ & $3.6 \pm 0.3$ & $3.3 \pm 0.3$ & & & \\
\hline $\mathrm{Ar}$ & $6.7 \pm 0.3$ & $7.1 \pm 0.4$ & $7.7 \pm 0.4$ & $7.5 \pm 0.5$ & & & \\
\hline $\mathrm{K}$ & $4.9 \pm 0.2$ & $5.3 \pm 0.3$ & $5.5 \pm 0.3$ & $5.9 \pm 0.4$ & & & \\
\hline $\mathrm{Ca}$ & $14.7 \pm 0.5$ & $15.1 \pm 0.6$ & $15.7 \pm 0.7$ & $16.0 \pm 0.9$ & $14.7 \pm 0.9$ & & \\
\hline $\mathrm{Sc}$ & $2.9 \pm 0.2$ & $3.1 \pm 0.2$ & $3.4 \pm 0.2$ & $3.1 \pm 0.2$ & $3.0 \pm 0.3$ & & \\
\hline $\mathrm{Ti}$ & $11.5 \pm 0.4$ & $12.5 \pm 0.6$ & $12.2 \pm 0.6$ & $12.0 \pm 0.7$ & $11.5 \pm 0.8$ & & \\
\hline $\mathrm{V}$ & $5.4 \pm 0.3$ & $5.4 \pm 0.3$ & $5.3 \pm 0.3$ & $5.7 \pm 0.4$ & $5.3 \pm 0.4$ & & \\
\hline $\mathrm{Cr}$ & $11.2 \pm 0.5$ & $11.3 \pm 0.5$ & $11.4 \pm 0.6$ & $11.3 \pm 0.6$ & $10.7 \pm 0.7$ & & \\
\hline Mn & $6.8 \pm 0.3$ & $7.1 \pm 0.3$ & $7.2 \pm 0.4$ & $6.9 \pm 0.4$ & $6.4 \pm 0.5$ & & \\
\hline $\mathrm{Fe}$ & $73.4 \pm 2.5$ & $73.9 \pm 2.8$ & $72.1 \pm 2.8$ & $68.7 \pm 3.4$ & $64.0 \pm 3.6$ & $58.3 \pm 3.6$ & \\
\hline Co & $0.31 \pm 0.05$ & $0.36 \pm 0.04$ & $0.40 \pm 0.05$ & $0.36 \pm 0.06$ & $0.40 \pm 0.07$ & $0.34 \pm 0.07$ & \\
\hline $\mathrm{Ni}$ & $3.1 \pm 0.2$ & $3.3 \pm 0.2$ & $3.5 \pm 0.2$ & $3.5 \pm 0.3$ & $3.1 \pm 0.2$ & $3.0 \pm 0.3$ & \\
\hline
\end{tabular}

Note. The systematic and statistical uncertainties are combined in quadrature.

The fourth term, with mean free path $\Lambda_{\text {esc }}^{i}$, represents cosmicray escape from the Galaxy. This energy-dependent quantity is empirically determined based on studies of secondary-toprimary ratios (e.g., Davis et al. 2000).

The final term on the right, in which $w_{i} \equiv(\mathrm{d} \varepsilon / \mathrm{d} x)_{i}$ is the specific ionization per nucleon in the interstellar medium, accounts for spectral changes due to ionization energy loss. The model does not include energy changes due to reacceleration processes; studies by Heinbach \& Simon (1995) and Scott (2005) indicate that such effects are relatively minor at the interstellar energies of the particles we are considering. Some studies have also shown that a small amount of reacceleration can reproduce the spectral shape at low energies (Shibata et al. 2006, and references therein), though we find the same features are reproducible using an energy-dependent leaky box escape length.

Source spectra were taken to be power laws in momentum with a spectral index of -2.35 . Solar system values from Lodders (2003) were assumed for the isotopic composition of each element in the source, except in the case of neon 
Table B4

CRIS Solar Maximum Spectra Interpolated to a Common Energy Grid

\begin{tabular}{|c|c|c|c|c|c|c|c|}
\hline \multicolumn{8}{|c|}{$\begin{array}{c}\text { Energies }(\mathrm{MeV} \text { nucleon } \\
\text { Intensities }\left(10^{-9}\left(\mathrm{~cm}^{2} \mathrm{~s} \text { sr MeV nucleon }{ }^{-1}\right)^{-1}\right) \\
\text { In }\end{array}$} \\
\hline Element & 60 & 72 & 85 & 100 & 120 & 142 & 170 \\
\hline $\mathrm{B}$ & $29.5 \pm 1.0$ & $33.6 \pm 1.0$ & $37.9 \pm 1.2$ & $42.6 \pm 1.4$ & $48.8 \pm 1.7$ & $54.3 \pm 2.0$ & $58.1 \pm 2.5$ \\
\hline $\mathrm{C}$ & & $113.2 \pm 3.3$ & $127.3 \pm 3.8$ & $143.8 \pm 4.3$ & $165.6 \pm 5.2$ & $184.9 \pm 6.2$ & $205.3 \pm 7.8$ \\
\hline $\mathrm{N}$ & & $30.7 \pm 1.0$ & $34.1 \pm 1.1$ & $38.0 \pm 1.2$ & $44.5 \pm 1.5$ & $50.2 \pm 1.7$ & $56.0 \pm 2.2$ \\
\hline $\mathrm{O}$ & & & $126.2 \pm 3.7$ & $140.1 \pm 4.2$ & $159.5 \pm 4.8$ & $179.8 \pm 5.7$ & $195.3 \pm 6.7$ \\
\hline $\mathrm{F}$ & & & $2.4 \pm 0.1$ & $2.7 \pm 0.1$ & $3.1 \pm 0.1$ & $3.3 \pm 0.2$ & $3.7 \pm 0.2$ \\
\hline $\mathrm{Ne}$ & & & $19.8 \pm 0.7$ & $22.3 \pm 0.7$ & $25.5 \pm 0.8$ & $28.3 \pm 1.0$ & $31.2 \pm 1.1$ \\
\hline $\mathrm{Na}$ & & & & $4.7 \pm 0.2$ & $5.2 \pm 0.2$ & $5.8 \pm 0.2$ & $6.4 \pm 0.3$ \\
\hline $\mathrm{Mg}$ & & & & $29.0 \pm 0.9$ & $32.8 \pm 1.0$ & $36.8 \pm 1.2$ & $41.2 \pm 1.4$ \\
\hline $\mathrm{Al}$ & & & & $4.6 \pm 0.2$ & $5.3 \pm 0.2$ & $6.0 \pm 0.2$ & $6.8 \pm 0.3$ \\
\hline $\mathrm{Si}$ & & & & & $24.5 \pm 0.8$ & $27.1 \pm 0.9$ & $30.0 \pm 1.0$ \\
\hline $\mathrm{P}$ & & & & & $0.8 \pm 0.1$ & $1.0 \pm 0.1$ & $1.1 \pm 0.1$ \\
\hline $\mathrm{S}$ & & & & & $4.3 \pm 0.2$ & $4.8 \pm 0.2$ & $5.4 \pm 0.2$ \\
\hline $\mathrm{Cl}$ & & & & & $0.9 \pm 0.1$ & $1.0 \pm 0.1$ & $1.1 \pm 0.1$ \\
\hline $\mathrm{Ar}$ & & & & & $1.9 \pm 0.1$ & $2.0 \pm 0.1$ & $2.3 \pm 0.1$ \\
\hline $\mathrm{K}$ & & & & & & $1.7 \pm 0.1$ & $1.8 \pm 0.1$ \\
\hline $\mathrm{Ca}$ & & & & & & $4.2 \pm 0.2$ & $4.8 \pm 0.2$ \\
\hline Sc & & & & & & $0.9 \pm 0.1$ & $1.1 \pm 0.1$ \\
\hline $\mathrm{Ti}$ & & & & & & $3.4 \pm 0.1$ & $3.8 \pm 0.1$ \\
\hline $\mathrm{V}$ & & & & & & $1.5 \pm 0.1$ & $1.6 \pm 0.1$ \\
\hline $\mathrm{Cr}$ & & & & & & $2.9 \pm 0.1$ & $3.3 \pm 0.1$ \\
\hline $\mathrm{Mn}$ & & & & & & $2.0 \pm 0.1$ & $2.1 \pm 0.1$ \\
\hline $\mathrm{Fe}$ & & & & & & & $22.1 \pm 0.8$ \\
\hline $\mathrm{Co}$ & & & & & & & $0.10 \pm 0.01$ \\
\hline $\mathrm{Ni}$ & & & & & & & $1.0 \pm 0.1$ \\
\hline Element & 200 & 240 & 285 & 340 & 400 & 475 & \\
\hline $\mathrm{C}$ & $218.1 \pm 8.9$ & & & & & & \\
\hline $\mathrm{N}$ & $59.9 \pm 2.6$ & & & & & & \\
\hline $\mathrm{O}$ & $212.5 \pm 8.5$ & $221.3 \pm 9.7$ & & & & & \\
\hline $\mathrm{F}$ & $4.0 \pm 0.2$ & $4.4 \pm 0.3$ & & & & & \\
\hline $\mathrm{Ne}$ & $34.6 \pm 1.4$ & $37.1 \pm 1.6$ & & & & & \\
\hline $\mathrm{Na}$ & $6.7 \pm 0.3$ & $7.5 \pm 0.4$ & $7.9 \pm 0.4$ & & & & \\
\hline $\mathrm{Mg}$ & $44.4 \pm 1.6$ & $47.8 \pm 2.0$ & $49.6 \pm 2.4$ & & & & \\
\hline $\mathrm{Al}$ & $7.5 \pm 0.3$ & $8.2 \pm 0.4$ & $8.1 \pm 0.4$ & & & & \\
\hline $\mathrm{Si}$ & $32.4 \pm 1.1$ & $34.7 \pm 1.3$ & $37.9 \pm 1.8$ & & & & \\
\hline $\mathrm{P}$ & $1.1 \pm 0.1$ & $1.3 \pm 0.1$ & $1.5 \pm 0.1$ & $1.6 \pm 0.1$ & & & \\
\hline $\mathrm{S}$ & $6.1 \pm 0.2$ & $6.8 \pm 0.3$ & $6.8 \pm 0.3$ & $7.5 \pm 0.4$ & & & \\
\hline $\mathrm{Cl}$ & $1.3 \pm 0.1$ & $1.4 \pm 0.1$ & $1.5 \pm 0.1$ & $1.8 \pm 0.1$ & & & \\
\hline $\mathrm{Ar}$ & $2.6 \pm 0.1$ & $3.0 \pm 0.1$ & $3.2 \pm 0.2$ & $3.3 \pm 0.2$ & & & \\
\hline $\mathrm{K}$ & $2.0 \pm 0.1$ & $2.3 \pm 0.1$ & $2.5 \pm 0.1$ & $2.6 \pm 0.2$ & & & \\
\hline $\mathrm{Ca}$ & $5.2 \pm 0.2$ & $5.5 \pm 0.2$ & $6.0 \pm 0.3$ & $6.5 \pm 0.4$ & $6.6 \pm 0.4$ & & \\
\hline $\mathrm{Sc}$ & $1.2 \pm 0.1$ & $1.2 \pm 0.1$ & $1.3 \pm 0.1$ & $1.4 \pm 0.1$ & $1.4 \pm 0.1$ & & \\
\hline $\mathrm{Ti}$ & $4.1 \pm 0.2$ & $4.3 \pm 0.2$ & $4.5 \pm 0.2$ & $4.6 \pm 0.3$ & $4.6 \pm 0.3$ & & \\
\hline $\mathrm{V}$ & $1.8 \pm 0.1$ & $1.9 \pm 0.1$ & $2.1 \pm 0.1$ & $2.2 \pm 0.1$ & $2.1 \pm 0.2$ & & \\
\hline $\mathrm{Cr}$ & $3.7 \pm 0.1$ & $3.9 \pm 0.2$ & $4.0 \pm 0.2$ & $4.3 \pm 0.2$ & $4.4 \pm 0.3$ & & \\
\hline $\mathrm{Mn}$ & $2.1 \pm 0.1$ & $2.5 \pm 0.1$ & $2.6 \pm 0.1$ & $2.7 \pm 0.2$ & $2.8 \pm 0.2$ & & \\
\hline $\mathrm{Fe}$ & $23.5 \pm 0.8$ & $25.3 \pm 1.0$ & $26.7 \pm 1.0$ & $27.8 \pm 1.4$ & $28.4 \pm 1.6$ & $27.7 \pm 1.7$ & \\
\hline Co & $0.13 \pm 0.02$ & $0.16 \pm 0.02$ & $0.18 \pm 0.02$ & $0.17 \pm 0.02$ & $0.19 \pm 0.02$ & $0.19 \pm 0.03$ & \\
\hline $\mathrm{Ni}$ & $1.1 \pm 0.1$ & $1.2 \pm 0.1$ & $1.3 \pm 0.1$ & $1.4 \pm 0.1$ & $1.6 \pm 0.1$ & $1.4 \pm 0.1$ & \\
\hline
\end{tabular}

Note. The systematic and statistical uncertainties are combined in quadrature.

where the ${ }^{22} \mathrm{Ne} /{ }^{20} \mathrm{Ne}$ source ratio derived from CRIS data (Binns et al. 2005) was used. Source elemental abundances for those elements containing a significant fraction of primary material (i.e., $\mathrm{C}-\mathrm{O}, \mathrm{Ne}-\mathrm{Si}, \mathrm{S}, \mathrm{Ar}, \mathrm{Ca}, \mathrm{Fe}-\mathrm{Ni}$ ) were adjusted to reproduce the measurements at Earth. For dominantly secondary elements, solar values relative to a nearby primary element with comparable first ionization potential were assumed. The interstellar medium was assumed to contain 0.34 hydrogen atoms $\mathrm{cm}^{-3}$ (Yanasak et al. 2001) and to have $\mathrm{He} / \mathrm{H}=0.11$ by number. Increased energy loss due to the presence of ionized hydrogen was handled by increasing the energy loss in hydrogen by a factor of 1.4 as suggested by Soutoul et al. (1990).

The mean free path for escape from the Galaxy was taken to have the form given by Davis et al. (2000). The other required mean free paths were calculated using interaction or decay data obtained from laboratory measurements or from calculations. Total spallation cross sections were based on Letaw et al. (1983) and Tripathi et al. (1997). Radioactive isotopes that have halflives for $\beta^{ \pm}$-decay at least as long as that of ${ }^{14} \mathrm{C}(5730 \mathrm{yr})$ or that decay only by electron capture were treated explicitly using 
Equation (C1). Nuclides that $\beta$-decay with shorter half-lives were assumed to decay immediately upon production and cross sections for producing their daughter products were adjusted accordingly. Half-lives of electron-capture nuclides having a single orbital electron attached were adjusted (lengthened by a factor of $\sim 2$ ) from their laboratory values. Nuclides that can decay only by electron capture are effectively stable; decays are possible only after attaching an electron from the medium through which they are propagating.

Partial cross sections for production of individual nuclides by spallation of heavier nuclei on interstellar hydrogen were based on the semiempirical formulae of Silberberg et al. (1998) using the yieldx_080999. for version of their code. The semiempirical results for individual reactions were renormalized (without adjusting the energy dependence) using measured cross sections when they were available in the energy range relevant to the present study. Cross sections for spallation on helium were scaled from the cross sections on hydrogen based on the work of Hirzebruch et al. (1993) and Kox et al. (1987). It is assumed in Equation $(\mathrm{C} 1)$ that spallation products are produced with the same velocity as the interacting projectile nucleus.

Cross sections for attachment and stripping of an orbital electron were taken from Crawford (1979) and Wilson (1978). Several typographical errors in these works were corrected in the current calculation. The calculated nonradiative attachment cross sections, which should have little effect for the energies and atomic numbers considered here, were reduced by a factor of 0.4 to reflect the experimental results (Crawford 1979).

Equation (C1) represents a system of ordinary, first-order differential equations, for the equilibrium interstellar intensities, $\varphi_{i}$, as a function of the energy per nucleon, $\varepsilon$, one for each ion being considered. Converting the independent variable to $\ln (\varepsilon)$ and using finite-difference techniques, we converted each of these equations into a tridiagonal matrix equation that can be solved numerically by standard techniques (e.g., Press et al. 1992) to yield $\varphi_{i}(\varepsilon)$. The set of the equations was solved progressing from heavier to lighter nuclides (and from lower to higher atomic number for nuclides of the same mass) so that the spallation production terms can be evaluated. In the cases of $\beta^{+}$and electron-capture decays, daughter nuclides are produced that occurred earlier in this ordering. These "backward" transitions are handled with a series of iteration loops over a limited set of affected nuclides, recalculating the abundances until a stable solution is obtained.

The calculated interstellar spectra for $\mathrm{C}, \mathrm{O}, \mathrm{Si}$, and $\mathrm{Fe}$ are shown with dashed lines in Figure 9. These spectra can be approximated with an analytic function of the form suggested by Webber \& Higbie (2003):

$$
\varphi(\varepsilon)=\frac{\varphi_{o}\left(\varepsilon / 10^{4} \mathrm{MeVnuc}^{-1}\right)^{\gamma_{o}}}{\left[1+\left(\varepsilon / \varepsilon_{1}\right)^{\alpha_{1}}+\left(\varepsilon / \varepsilon_{2}\right)^{\alpha_{2}}\right]} .
$$

This function, evaluated using the parameters listed in Table $\mathrm{C} 1$, reproduces the numerical results from the propagation calculation to within $10 \%$ over the energy range 50 $10^{4} \mathrm{MeV}_{\text {nucleon }}{ }^{-1}$. The accuracy of the approximation degrades rapidly outside this range. Furthermore, it should be kept in mind that below a few hundred $\mathrm{MeV}$ nucleon $^{-1}$ the interstellar spectra are, at best, poorly constrained by the modulated spectra measured near Earth.

\section{C.2. Solar Modulation}

Once equilibrium interstellar spectra have been derived, the effects of solar modulation are calculated from a spherically
Table C1

Parameters of Interstellar Spectra Fits for $50-10^{4} \mathrm{MeV}^{\text {Nucleon }}{ }^{-1}$

\begin{tabular}{lcccc}
\hline \hline \multicolumn{1}{c}{ Element } & $\mathrm{C}$ & $\mathrm{O}$ & $\mathrm{Si}$ & $\mathrm{Fe}$ \\
\hline$\varphi_{o}\left(\times 10^{10}\left(\mathrm{~cm}^{2} \mathrm{sr} \mathrm{s} \mathrm{MeV} \mathrm{nucleon}^{-1}\right)^{-1}\right.$ & 42.6 & 45.5 & 7.62 & 5.34 \\
$\gamma_{o}$ & -2.32 & -2.27 & -2.22 & -2.19 \\
$\varepsilon_{1}\left(\mathrm{MeV}_{\text {nucleon }}{ }^{-1}\right)$ & 868 & 857 & 858 & 900 \\
$\alpha_{1}$ & -1.71 & -1.71 & -1.75 & -1.46 \\
$\varepsilon_{2}\left(\mathrm{MeV}_{\text {nucleon }}{ }^{-1}\right)$ & 305 & 273 & 245 & 342 \\
$\alpha_{2}$ & -3.66 & -3.66 & -3.66 & -2.91 \\
\hline
\end{tabular}

symmetric model including diffusion, convection, and adiabatic deceleration using the Crank-Nicholson technique, as discussed by Fisk (1971). The interplanetary diffusion coefficient is taken to be of the form $\kappa(R)=\kappa_{0} \beta R / R_{0}$, where $\beta$ is the ratio of the particle speed to the speed of light, $R$ is the particle rigidity, and $\kappa_{0}$ sets the absolute value of the diffusion coefficient at a selected value of rigidity, $R_{0}$. The calculation also depends on the solar wind velocity, $V_{\mathrm{SW}}$, and the radius of the heliospheric modulation boundary, $D$. It is convenient to specify the level of modulation in terms of the parameter $\phi$ (measured in MV) introduced by Gleeson \& Axford (1968) in connection with the approximate "force field solution" of the spherically symmetric modulation equation (although we are not using that approximation for the calculations presented here)

$$
\phi \equiv \frac{R}{3} \int_{1 \mathrm{AU}}^{D} \frac{V_{\mathrm{SW}}(r)}{\kappa(r, R) / \beta} d r .
$$

With our assumptions that $\kappa \propto \beta R$ and that $\kappa$ and $V_{\mathrm{SW}}$ are independent of radius in the heliosphere, this reduces to $\phi=R_{0} V_{\mathrm{SW}}(D-1 \mathrm{AU}) / 3 \kappa_{0}$. Note that, to good accuracy, the calculated $1 \mathrm{AU}$ spectra depend on $V_{\mathrm{SW}}, \kappa_{0}$, and $D$ only in the combination $V_{\mathrm{SW}}(D-1 \mathrm{AU}) / \kappa_{0}$, provided that $D \gg 1 \mathrm{AU}$, as in the heliosphere. Typical values of $\phi$ for the solar minimum $(\phi \simeq 325 \mathrm{MV})$ and solar maximum $(\phi \simeq 900 \mathrm{MV})$ time periods used in the present study were estimated using results from Wiedenbeck et al. (2005) in which the time variation of the modulation level was derived from the measured shapes of elemental energy spectra (see Davis et al. 2001a, 2001b).

\section{REFERENCES}

Ahlen, S. P. 1980, Rev. Mod. Phys., 52, 121

Andersen, H. H., \& Ziegler, J. F. 1977, The Stopping Powers and Ranges of Ions in Matter, Vol. 3 (New York: Pergammon)

Binns, W. R., et al. 2005, ApJ, 634, 351

Crawford, H. J. 1979, PhD thesis, Univ. of California, Berkeley (LBL-8807)

Davis, A. J., et al. 2000, in AIP Conf. Ser. 528, Acceleration and Transport of Energetic Particles Observed in the Heliosphere, ed. R. A. Mewaldt et al. (Melville, NY: AIP), 421

Davis, A. J., et al. 2001a, J. Geophys. Res., 106, 29979

Davis, A. J., et al. 2001b, Proc. 27th Int. Cosmic Ray Conf. (Hamburg), 10, 3971

de Nolfo, G. A., et al. 2006, Adv. Space Res., 38, 1558

Derrickson, J. H., Parnell, T. A., Austin, R. W., Selig, W. J., \& Gregory, J. C. 1992, Int. J. Radiat. Appl. Instrum. D, 20, 415

DuVernois, M. A., \& Thayer, M. R. 1996, ApJ, 465, 982

Engelmann, J. J., et al. 1990, A\&A, 233, 96

Fisk, L. A. 1971, J. Geophys. Res., 76, 221

Garcia-Muñoz, M., Mason, G. M., Simpson, J. A., \& Wefel, J. P. 1977a, Proc. 15th Int. Cosmic Ray Conf. (Plovdiv), 1, 224

Garcia-Muñoz, M., Mason, G. M., Simpson, J. A., \& Wefel, J. P. 1977b, Proc 15th Int. Cosmic Ray Conf. (Plovdiv), 1, 230

Garcia-Muñoz, M., \& Simpson, J. A. 1979, Proc. 16th Int. Cosmic Ray Conf. (Kyoto), 1, 270

Gleeson, L. J., \& Axford, W. I. 1968, ApJ, 154, 1011

Heinbach, U., \& Simon, M. 1995, ApJ, 441, 209 
Hirzebruch, S. E., Winkel, E., \& Heinrich, W. 1993, Proc. 23rd Int. Cosmic Ray Conf. (Calgary), 2, 175

Kox, S., et al. 1987, Phys. Rev. C, 35, 1678

Letaw, J. R., Silberberg, R., \& Tsao, C. H. 1983, ApJS, 51, 271

Lezniak, J. A., \& Webber, W. R. 1978, ApJ, 223, 676

Lodders, K. 2003, ApJ, 591, 1220

Meneguzzi, M., Audouze, J., \& Reeves, H. 1971, A\&A, 15, 337

Mewaldt, R. A., et al. 2005, Proc. 29th Int. Cosmic Ray Conf. (Mumbai), 2, 433

Niebur, S. M., et al. 2003, J. Geophys. Res., 108, A10, 8033

O’Neill, P. M. 2006, Adv. Space Res., 37, 1727

Potgieter, M. S., \& Langner, U. W. 2004, Ann. Geophys., 22, 3729

Press, W. H., et al. 1992, Numerical Recipes in C (2nd ed.; Cambridge: Cambridge Univ. Press)

Scott, L. M. 2005, PhD thesis, Washington Univ., St. Louis

Shibata, T., Hareyama, M., Nakazawa, M., \& Saito, C. 2006, ApJ, 642, 882

Silberberg, R., Tsao, C. H., \& Barghouty, A. F. 1998, ApJ, 501, 911

Soutoul, A., Ferrando, P., \& Webber, W. R. 1990, Proc. 21st Int. Cosmic Ray Conf. (Adelaide), 3, 337
Stone, E. C., et al. 1998, Space Sci. Rev., 86, 285

Tripathi, R. K., Cucinotta, F. A., \& Wilson, J. W. 1997, Universal Parameterization of Absorption Cross Sections Tech. Rep. NASA, Technical Paper 3621

Usoskin, I. G., Alanko-Huotari, K., Kovaltsov, G. A., \& Mursula, K. 2005, J. Geophys. Res. (Space Phys.), 12108, 110

Wang, J. Z., et al. 2002, ApJ, 564, 244

Webber, W. R., \& Higbie, P. R. 2003, J. Geophys. Res. (Space Phys.), 1355,108

Westfall, G. D., Wilson, L. W., Lindstrom, P. J., Crawford, D. E., \& Heckman, H. H. 1979, Phys. Rev. C, 1309, 19

Wiedenbeck, M. E., et al. 2001, Space Sci. Rev., 99, 15

Wiedenbeck, M. E., et al. 2005, Proc. 29th Int. Cosmic Ray Conf. (Mumbai), 2, 277

Wilson, L. W. 1978, PhD thesis, Lawrence Berkeley Laboratory (Report No. LBL-7723)

Yanasak, N. E., et al. 2001, ApJ, 563, 768

Young, J. S., Freier, P. S., Waddington, C. J., Brewster, N. R., \& Fickle, R. K. 1981, ApJ, 246, 1014 